\title{
Finite-size effects in Luther-Emery phases of Holstein and Hubbard models
}

\author{
J. Greitemann, ${ }^{1,2}$ S. Hesselmann, ${ }^{2}$ S. Wessel, ${ }^{2}$ F. F. Assaad,${ }^{3}$ and M. Hohenadler ${ }^{3}$ \\ ${ }^{1}$ Department of Physics, Arnold Sommerfeld Center for Theoretical Physics and Center for NanoScience, \\ Ludwig-Maximilians-Universität München, 80333 Munich, Germany \\ ${ }^{2}$ Institute for Theoretical Solid State Physics, JARA-FIT, \\ and JARA-HPC, RWTH Aachen University, 52056 Aachen, Germany \\ ${ }^{3}$ Institut für Theoretische Physik und Astrophysik, Universität Würzburg, 97074 Würzburg, Germany
}

(Dated: October 11, 2018)

\begin{abstract}
The one-dimensional Holstein model and its generalizations have been studied extensively to understand the effects of electron-phonon interaction. The half-filled case is of particular interest, as it describes a transition from a metallic phase with a spin gap due to attractive backscattering to a Peierls insulator with charge-density-wave (CDW) order. Our quantum Monte Carlo results support the existence of a metallic phase with dominant power-law charge correlations, as described by the Luther-Emery fixed point. We demonstrate that for Holstein and also for purely fermionic models the spin gap significantly complicates finite-size numerical studies, and explains inconsistent previous results for Luttinger parameters and phase boundaries. On the other hand, no such complications arise in spinless models. The correct low-energy theory of the spinful Holstein model is argued to be that of singlet bipolarons with a repulsive, mutual interaction. This picture naturally explains the existence of a metallic phase, but also implies that gapless Luttinger liquid theory is not applicable.
\end{abstract}

PACS numbers: 71.30.+h, 02.70.Ss, 64.60.an, 71.10.Pm

\section{INTRODUCTION}

The interaction of charge carriers with the crystal lattice is a fundamental ingredient for the description of materials. In addition to its role for superconductivity, electron-phonon coupling manifests itself in phenomena such as the Peierls transition [1] and polaron formation [2]. Electron-phonon coupling appears to play an important role in a number of strongly correlated materials such as the manganites [3]. From a theoretical point of view, even a single electron coupled to quantum phonons represents a complex many-body problem [4], and systems with a finite band filling are even more demanding.

Much of our knowledge about the effects of electronphonon coupling in low-dimensional systems comes from investigations of simplified microscopic models. Among these models, the Holstein model [5] and its generalizations play a central role. The focus on Holstein-type models is a consequence of its relative simplicity, namely a density-displacement coupling and Einstein phonons, which makes the models amenable to exact numerical methods such as quantum Monte Carlo (QMC) or the density-matrix renormalization group (DMRG); for a review of existing work see Ref. 6 . In the one-dimensional (1D) case considered here, Holstein-type models have also been studied with the bosonization method in combination with the renormalization group (RG) $[7,8]$.

A critical look at the available literature reveals that, despite the substantial body of results, the understanding of the half-filled Holstein and Holstein-Hubbard models is incomplete. Apart from discrepancies in the critical values obtained with different numerical methods, there is a long-standing argument regarding the existence of a metallic phase. Whereas more recent large-scale numerical studies [9-12] support an extended metallic re- gion as a result of quantum lattice fluctuations, similar to the spinless Holstein model [13-15], earlier numerical and analytical results imply an insulating ground state for any nonzero electron-phonon coupling $[7,8,16]$. A recent functional RG study confirmed the existence of an extended metallic region [17]. Complications also arise when trying to understand numerical results in terms of Luttinger liquid theory. Most notably, the numerically determined values of the Luttinger parameter $K_{\rho}$ [9-11] conflict with the observed behavior of the correlation functions $[6,18-21]$. The situation is further complicated by the fact that the electron-phonon interaction gives rise to a spin gap [6], so that the low-energy physics is described by the Luther-Emery fixed point [22].

Here, we reveal the key role of the spin degrees of freedom for the observed inconsistencies. In addition to surveying previous results, we present new results for Holstein and purely fermionic models. A comparison between spinful and spinless Holstein models allows us to separate the effects of backscattering from retardation effects related to the lattice dynamics. On the other hand, transitions from a Luttinger or Luther-Emery liquid to a CDW phase can also be investigated in numerically more accessible fermionic models, for which a quantitative comparison between theory and simulation is possible. The central findings of this work are as follows. (i) Previous claims for the absence of a metallic phase [16] are shown to be unfounded. Instead, the spinful Holstein model seems to support a Peierls transition at a nonzero critical electron-phonon coupling. (ii) The metallic phase of the spinful Holstein model is characterized by powerlaw (dominant) charge and (subdominant) pairing correlations, and exponentially suppressed spin correlations. (iii) The spin gap caused by backscattering lies at the heart of the conflicts between numerics and theory. In 
particular, the expected Luther-Emery behavior is only observed on length scales larger than the inverse spin gap. (iv) The pairing of electrons into spin singlets (bipolarons) provides a connection to the physics of the better understood spinless Holstein model, and suggests that the correct low-energy picture of the Holstein model is that of hard-core bosons with repulsive interactions. The latter problem can be solved exactly, and provides a natural explanation for the existence of an extended metallic region in the Holstein model.

The paper is organized as follows. In Sec. II, we introduce the relevant models. The QMC methods are briefly explained in Sec. III. In Sec. IV, we discuss the spinful and spinless Holstein models, including correlation functions, Luttinger parameters, and the charge susceptibility. In Sec. V, we reveal important similarities with suitable fermionic models that capture the metal-insulator transition. Section VI gives a discussion of our and previous results and the correct low-energy theory of the Holstein model. Finally, Section VII contains our conclusions, and the Appendix gives the derivation of the scaling behavior of the charge susceptibility.

\section{MODELS}

The 1D Holstein model is defined as [5]

$$
\begin{aligned}
\hat{H}= & -t \sum_{i \sigma}\left(c_{i \sigma}^{\dagger} c_{i+1 \sigma}+\text { H.c. }\right) \\
& +\sum_{i}\left(\frac{1}{2 M} \hat{P}_{i}^{2}+\frac{K}{2} \hat{Q}_{i}^{2}\right)-g \sum_{i} \hat{Q}_{i}\left(\hat{n}_{i}-1\right) .
\end{aligned}
$$

The first term describes the hopping of electrons between neighboring lattice sites with amplitude $t$. The second term describes the lattice degrees of freedom in the harmonic approximation; the phonon frequency is given by $\omega_{0}=\sqrt{K / M}, \hat{Q}_{i}\left(\hat{P}_{i}\right)$ is the lattice displacement (momentum) at site $i$. The electron-phonon interaction described by the third term couples the electron density at site $i$ with the lattice distortion at the same site. The electron density operator is defined as $\hat{n}_{i \sigma}=c_{i \sigma}^{\dagger} c_{i \sigma}$, and we have $\hat{n}_{i}=\sum_{\sigma} \hat{n}_{i \sigma}$. The dimensionless parameter $\lambda=g^{2} /(4 K t)$ is a useful measure for the electronphonon coupling strength. A common alternative notation for the interaction term is $-\bar{g} \sum_{i}\left(b_{i}^{\dagger}+b_{i}\right)\left(\hat{n}_{i}-1\right)$ where $g=\bar{g} \sqrt{2 M \omega_{0}}$ and $\lambda=\bar{g}^{2} /\left(2 \omega_{0}\right)$. Equation (1) has been generalized to the Holstein-Hubbard model [23] with an additional repulsive interaction $U \sum_{i} \hat{n}_{i \uparrow} \hat{n}_{i \downarrow}$, see Ref. 6 for a review.

The spinless Holstein model [16]

$$
\begin{aligned}
\hat{H}= & -t \sum_{i}\left(c_{i}^{\dagger} c_{i+1}+\text { H.c. }\right) \\
& +\sum_{i}\left(\frac{1}{2 M} \hat{P}_{i}^{2}+\frac{K}{2} \hat{Q}_{i}^{2}\right)-g \sum_{i} \hat{Q}_{i}\left(\hat{n}_{i}-0.5\right),
\end{aligned}
$$

with $\hat{n}_{i}=c_{i}^{\dagger} c_{i}$, captures much of the physics of Eq. (1), and will provide important insights.
We further consider the $U-V$ extended Hubbard model

$$
\begin{aligned}
\hat{H}= & -t \sum_{i \sigma}\left(c_{i \sigma}^{\dagger} c_{i+1 \sigma}+\text { H.c. }\right) \\
& +U \sum_{i} \hat{n}_{i \uparrow} \hat{n}_{i \downarrow}+V \sum_{i} \hat{n}_{i} \hat{n}_{i+1}
\end{aligned}
$$

with onsite interaction $U$ and nearest-neighbor interaction $V$. In the nonadiabatic limit $\omega_{0} \rightarrow \infty$, the spinful Holstein model can be mapped to Eq. (3) with $U=-4 \lambda t<0[16]$ and $V=0$. More generally, Eq. (3) with $U<0$ describes a transition from a spin-gapped metallic phase at $V=0$ to a CDW insulator at $V>0$.

Finally, we investigated the spinless $t$ - $V$ model

$$
\hat{H}=-t \sum_{i}\left(c_{i}^{\dagger} c_{i+1}+\text { H.c. }\right)+V \sum_{i} \hat{n}_{i} \hat{n}_{i+1}
$$

which for half-filling is known to have a metal-insulator transition at $V=2 t$.

We studied the above models at half-filling, corresponding to $\langle n\rangle=1$ for Eqs. (1) and (3), and to $\langle n\rangle=0.5$ for Eqs. (2) and (4). We use $t$ as the unit of energy, and set the lattice constant and $\hbar$ to one.

\section{METHODS}

We simulated the models defined in Sec. II with two different QMC methods. First, we made use of the continuous-time interaction expansion (CT-INT) method [24] which has been successfully applied to electronphonon lattice models [25-28]. The phonons are integrated out analytically, and the resulting fermionic model with nonlocal (i.e., retarded) interactions is simulated [29]. We refer to previous publications [25-29] and reviews $[30,31]$ for technical details.

Second, we used the stochastic series expansion (SSE) representation [32], which in principle provides a more favorable linear (as compared to cubic for the CT-INT method) scaling of computer time with system size $L$ and inverse temperature $\beta=1 / k_{\mathrm{B}} T$. It was previously applied to the Holstein-Hubbard model [9, 10]. For the Holstein model, the phonons have been treated explicitly in the occupation number basis. In order to avoid negative vertex weights, a cut-off on the maximum phonon occupation needs to be imposed. However, unlike exact diagonalization or DMRG methods, the computational effort scales only linearly in the phonon cut-off, allowing us to choose it sufficiently large to make the resulting systematic errors completely negligible. While the employed update scheme is inherently grand-canonical, measurements were restricted to half-filled configurations [33].

In the SSE representation, the directed loops algorithm [32] allows us to update the purely fermionic models very efficiently and to reach significantly larger system sizes compared to the CT-INT method. In contrast, the phononic operators of the Holstein model are updated 
by exchanging pairs of phonon creation and annihilation operators on the same site with diagonal electronic operators, and vice versa, with Metropolis probabilities $[10,34]$. The number of diagonal phonon operators between the pair enters the acceptance probabilities as an exponent. Thus, in the nonadiabatic regime, $\omega_{0} \gg t$, the acceptance rates for phononic updates are exponentially suppressed, leading to prolonged autocorrelation times. This can be remedied to some extent by the use of quantum parallel tempering [35]. Still, for $\omega_{0} / t=5$ we found that the CT-INT method is in fact competitive.

We measured the real-space correlation functions in the charge, pairing, and spin channels,

$$
\begin{aligned}
S_{\rho}(r) & =\left\langle\left(\hat{n}_{r}-n\right)\left(\hat{n}_{0}-n\right)\right\rangle, \\
S_{\pi}(r) & =\left\langle\hat{\pi}_{r}^{\dagger} \hat{\pi}_{0}\right\rangle, \\
S_{\sigma}^{\alpha}(r) & =\left\langle\hat{S}_{r}^{\alpha} \hat{S}_{0}^{\alpha}\right\rangle .
\end{aligned}
$$

Here $\alpha=x, z$, and the pairing operator is given by $\hat{\pi}_{r}^{\dagger}=c_{r \uparrow}^{\dagger} c_{r \downarrow}^{\dagger}$ in the spinful case, and by $\pi_{r}^{\dagger}=c_{r}^{\dagger} c_{r+1}^{\dagger}$ in the spinless case. We also consider the corresponding structure factors obtained via Fourier transformation,

$$
S_{\nu}^{(\alpha)}(q)=\sum_{r} e^{\mathrm{i} q r} S_{\nu}^{(\alpha)}(r) .
$$

\section{HOLSTEIN MODELS}

\section{A. Overview of existing work}

We begin with an overview of important previous results for the half-filled Holstein model. The reason for focusing on the pure Holstein model is that it features only one phase transition, whereas the Holstein-Hubbard model exhibits Mott and Peierls transitions [6, 10, 11].

\section{Numerical results}

A variety of numerical methods have been applied to the half-filled Holstein model, most notably exact diagonalization [36-38] and QMC methods [6, 9, 10, 16, 19], as well as the DMRG [11, 12, 21, 39]. The first study was carried out using a QMC algorithm [16]. Based on results for the real-space charge correlation function, and reassured by approximate analytical arguments, it was claimed that the spinful Holstein model at half-filling is a Peierls insulator for any finite phonon frequency $\omega_{0}<\infty$ [16]. We will show in Sec. IV B that this conclusion was incorrect. Accordingly, all ensuing numerical works suggest that the model instead has an extended metallic phase where quantum lattice fluctuations destroy the dimerized Peierls state below a critical value of the electron-phonon coupling $[10,11]$. The numerical phase diagrams $[10,11]$ agree with respect to the overall features but, as discussed below, there are nonnegligible differences regarding the phase boundaries. Despite these quantitative uncertainties, the DMRG and QMC results rather firmly establish the existence of a phase transition from a spin-gapped metallic phase to a charge-ordered insulating phase with increasing electron-phonon coupling.

Interest in the Holstein(-Hubbard) model revived when QMC simulations [10] suggested the existence of a metallic phase with dominant pairing correlations, as indicated by a Luttinger parameter $K_{\rho}>1$. However, it was soon shown that for $\lambda>0$ charge correlations always decay slower than pairing correlations [18]. This conflict between the numerical values of $K_{\rho}$ and the behavior of the correlation functions has not been resolved. An important property of the metallic phase is the existence of a spin gap, first pointed out in Ref. 10, which arises from the pairing of electrons into spin singlets. In the language of bosonization, the spin gap is caused by attractive backscattering of electrons, similar to the attractive Hubbard model. A detailed discussion of the spin gap can be found in Ref. 6. The most compelling evidence for a spin gap is the dominance of charge correlations over spin correlations, which is not possible in a gapless Luttinger liquid [40] but observed in the Holstein model in the entire metallic phase [6], as well as the numerical finding of a Luttinger parameter $K_{\sigma}<1$ incompatible with the $\mathrm{SU}(2)$ spin symmetry of the Holstein model [6] (see Sec. IV C). An important corollary of the existence of a spin gap in the metallic phase is that the relevant fixed point in the thermodynamic limit is not the Luttinger liquid but the Luther-Emery liquid, which is much more difficult to analyze.

\section{Analytical results}

In the static (classical phonon or adiabatic) limit $\omega_{0} \rightarrow$ 0 a mean-field ansatz $Q_{i}=(-1)^{i} \Delta$ reveals the Peierls instability inherent to $1 \mathrm{D}$ systems without quantum fluctuations. Because the energy gain outweighs the cost for dimerization, any nonzero electron-phonon coupling leads to an insulating Peierls state. This conclusion holds for both the spinful and the spinless Holstein model. In the opposite, anti-adiabatic limit $\omega_{0} \rightarrow \infty$, the Holstein model can be mapped to the attractive Hubbard model with an instantaneous interaction $U=-4 \lambda t$ [16]. The half-filled attractive Hubbard model is metallic for any $U$, and has a spin gap $\Delta_{\sigma} \sim e^{-v_{\mathrm{F}} / \mathcal{U}}$ where $\mathcal{U}$ is the effective backscattering matrix element [41]. While representing valuable limiting cases, neither the static $\left(\omega_{0} \rightarrow 0\right)$ nor the instantaneous $\left(\omega_{0} \rightarrow \infty\right)$ limit captures the theoretical problem of electrons coupled to quantum phonons.

The strong-coupling approximation presented by Hirsch and Fradkin [16] starts from electrons paired into spin singlets and considers the effect of second-order hopping processes to derive an effective hardcore boson model with pair hopping $\widetilde{t}$ and nearest-neighbor repulsion $\widetilde{V}$, both functions of $\lambda$ and $\omega_{0}$. The strong-coupling results give $\widetilde{V} / 2 \widetilde{t}>1$ for any $\omega_{0}<\infty$ which, according to 
the bosonization results for hardcore bosons, implies that the system is in an insulating, charge-ordered phase for any $\lambda>0$. In contrast, a similar approximation for the spinless Holstein model gives $\lambda_{c}>0$. [Interestingly, the same difference between the spinful and spinless cases is also predicted for the Su-Schrieffer-Heeger model. In contrast to the Holstein model, these analytical predictions $\left(\lambda_{c}>0\right.$ for the spinless model, $\lambda_{c}=0$ for the spinful model) have been confirmed by numerical simulations, see Ref. 42 and references therein.] The strong-coupling approximation highlights the relation of the spinful Holstein model to hardcore bosons (corresponding to electron pairs), but is considered to be unreliable in the weak-coupling regime. In particular, for sufficiently small $\lambda$, the approximation of electron pairs as hardcore, onsite objects breaks down. Instead, the system may be regarded as consisting of interacting singlet bipolarons whose size depends on $\lambda$ and $\omega_{0}$.

Because we are considering a $1 \mathrm{D}$ system, important insights can be gained from the bosonization method together with the RG. For electron-phonon models, such an approach involves an additional approximation. The RG flow is carried out in two steps: From high energies down to the energy for phonon excitations, then (assuming an instantaneous interaction) from the phonon energy down to zero $[7,8]$. Additionally, the momentum dependence of the phonon-mediated electron-electron interaction is usually neglected. For the spinful Holstein model, the RG suggests $\lambda_{c}=0$ for $\omega_{0}<\infty$, whereas $\lambda_{c}>0$ is found for the spinless Holstein model [7, 8]. The results for the spinful Holstein model are often quoted as evidence for the absence of a metallic phase, despite the abovementioned limitations. An extended metallic phase was recently confirmed using the functional RG method [17], which takes into account the frequency dependence of the interaction.

\section{B. Existence of a metallic phase}

To settle the argument, we revisit the QMC results of Hirsch and Fradkin (HF) [16], the only numerical results that suggest the absence of a metallic phase. Their conclusions rely on data for the real-space charge and lattice correlation functions [Figs. 7(b) and 8(b) in Ref. 16]. For the parameters considered, the data suggest long-range order (up to the system size considered) for charge and lattice correlations in the spinful Holstein model both at low and high phonon frequencies, from which the authors conclude the absence of a metallic phase. In contrast, a crossover from long-range to short-range order was observed in the spinless model upon increasing the phonon frequency, which is compatible with a Peierls transition as a function of $\omega_{0}$ or, equivalently, $\lambda$.

The coupling constant used in Ref. 16, denoted here as $\lambda_{\mathrm{HF}}$, is related to $\lambda$ via $\lambda=\lambda_{\mathrm{HF}}^{2} /(4 K t)$. HF set $t=1$ and $K=0.25$, so that $\lambda=\lambda_{\mathrm{HF}}^{2}$. They considered $\lambda_{\mathrm{HF}}=0.9$ $[\lambda=0.81]$ for the spinless model, and $\lambda_{\mathrm{HF}}=0.9 / \sqrt{2}[\lambda=$
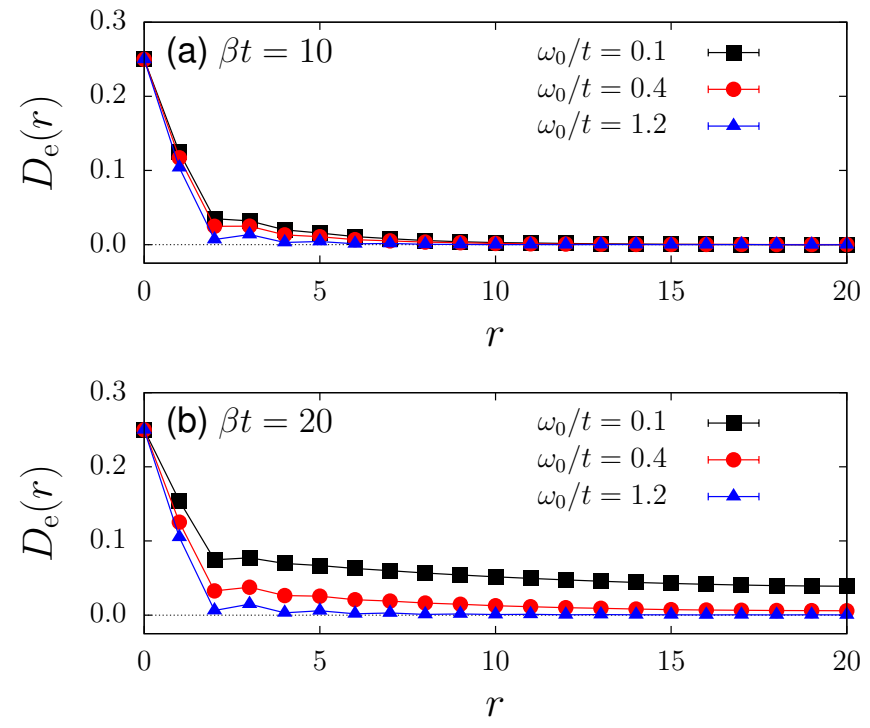

FIG. 1. (Color online) Charge correlations [Eq. (7)] for the spinless Holstein model at (a) $\beta t=10$, (b) $\beta t=20$. Here, $\lambda=$ 0.81 and $L=42$. The results can be compared to Fig. 7(b) in Ref. 16. Results obtained with the CT-INT method. Here and in subsequent figures, the error bars are typically smaller than the symbols.

$0.405]$ for the spinful model, as well as phonon frequencies $\omega_{0} / t=0.4$ and $\omega_{0} / t=1.2$. The inverse temperature was $\beta t=10$ for $\omega_{0} / t=0.4$ and $\beta t=5$ for $\omega_{0} / t=1.2$. The system size was $L=40$ sites. In contrast to the continuous-time methods used here, the results of Ref. 16 also have a systematic Trotter error.

Using the CT-INT method, we calculated the charge correlation function considered by HF [16],

$$
D_{\mathrm{e}}(r)=\frac{1}{L} \sum_{i \sigma \sigma^{\prime}}(-1)^{r}\left[\left\langle\hat{n}_{i \sigma} \hat{n}_{i+r, \sigma^{\prime}}\right\rangle-n^{2}\right],
$$

which is equivalent to $(-1)^{r} S_{\rho}(r)$, cf. Eq. (5).

Results for $D_{\mathrm{e}}(r)$ of the spinless model are shown in Fig. 1 for $L=42$. For the lowest temperature used by $\mathrm{HF}, \beta t=10$, Fig. 1 (a) reveals the absence of long-range charge correlations for all values of $\omega_{0}$ considered (in conflict with Ref. 16). At a lower temperature, $\beta t=20$, we find results similar to those of $\mathrm{HF}$, namely long-range order for $\omega_{0} / t=0.4$ but not for $\omega_{0} / t=1.2$. We attribute the discrepancies for $\beta t=10$ to autocorrelations, which can be significant for the parameters considered [43], thereby giving rise to spuriously enhanced charge correlations. Autocorrelations are significantly smaller and properly accounted for in the present results. The results in Fig. 1(b) are consistent with a Peierls transition as a function of phonon frequency in the spinless Holstein model, as claimed in Ref. 16.

Results for the spinful Holstein model are shown in Fig. 2. For parameters comparable to those used by HF, Fig. 2(a), we find long-range correlations up to the maximal distance for $\omega_{0} / t=0.4$, but not for $\omega_{0} / t=1.2$. 

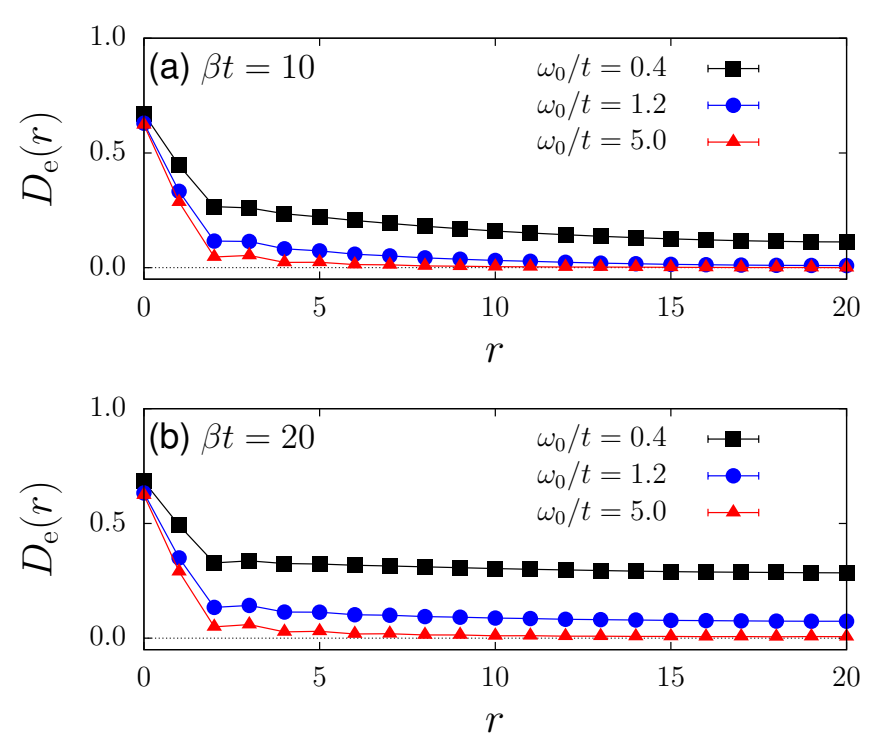

FIG. 2. (Color online) Charge correlations [Eq. (7)] for the spinful Holstein model at (a) $\beta t=10$, (b) $\beta t=20$. Here, $\lambda=$ 0.405 and $L=42$. The results can be compared to Fig. 8(b) in Ref. 16. Results obtained with the CT-INT method.

However, both cases show significantly weaker charge correlations than those in Ref. 16, a fact that we again attribute to autocorrelations. At a lower temperature, shown in Fig. 2(b), similar to the spinless case, we find results which look similar to those of $\mathrm{HF}$, namely longrange charge order for both $\omega_{0} / t=0.4$ and $\omega_{0} / t=1.2$. However, if we increase $\omega_{0}$ further, we again find a transition to a state without long-range order, consistent with a metallic state at $\lambda>0$.

To estimate $\lambda_{c}$, we carried out a finite-size extrapolation of the charge correlations at the largest distance. The results in Fig. 3 are consistent with extended metallic regions in both the spinless and the spinful model. We have verified that our low-temperature results are representative of the ground state. The onset of long-range order in Fig. 3 is consistent with the best available estimates for the critical values of the spinless $\left(\lambda_{c} \approx 0.39\right.$ [14]) and the spinful Holstein model $\left(\lambda_{c} \approx 0.23[9-12]\right)$.

\section{Real-space correlation functions}

In $1 \mathrm{D}$ metals, the decay of correlation functions is expected to be parametrized by the charge and spin Luttinger parameters $K_{\rho}$ and $K_{\sigma}$, respectively. However, previous numerical and analytical work revealed a number of difficulties in applying Luttinger liquid theory to the spinful Holstein model, whereas good agreement was observed for the spinless Holstein model [15]. Most importantly, the values of $K_{\rho}$ obtained with exact numerical methods are incompatible with the numerically determined correlation functions.
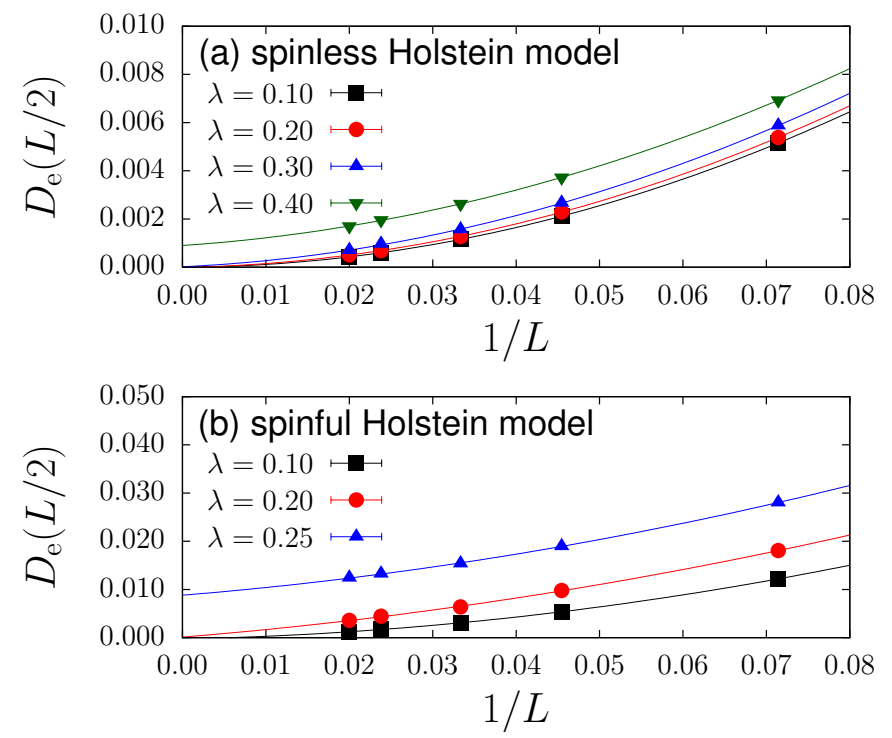

FIG. 3. (Color online) Finite-size scaling of the charge correlations at the largest distance $r=L / 2$. Lines are fits to second-order polynomials. Here, $\beta t=L$, (a) $\omega_{0} / t=0.1$, (b) $\omega_{0} / t=0.5$. Results obtained with the CT-INT method.

\section{Spinless Holstein model}

To set the stage for the discussion of the spinful case, it is useful to first consider the spinless Holstein model (2). The bosonization method applied to 1D spinless fermions gives the following results for the real-space correlation functions (we use $x$ to denote distances in the continuum limit, and $r$ for distances on a lattice) [44]:

$$
\begin{aligned}
S_{\rho}(x) & =-\frac{K_{\rho}}{2 \pi^{2} x^{2}}+\frac{A_{\rho}}{x^{2 K_{\rho}}} \cos \left(2 k_{\mathrm{F}} x\right), \\
S_{\pi}(x) & =\frac{A_{\pi}}{x^{2 K_{\rho}^{-1}}} .
\end{aligned}
$$

Here, $\rho(\pi)$ denotes the charge (pairing) sector.

According to these results, the exponents determining the power-law decay of correlations depend only on $K_{\rho}$. Equation (8) further implies that $q=0$ pairing correlations dominate (i.e., decay slowest) in the case of attractive interactions $\left(K_{\rho}>1\right)$, whereas $q=2 k_{\mathrm{F}}$ charge correlations dominate in the case of repulsive interactions $\left(K_{\rho}<1\right)$. For noninteracting electrons $\left(K_{\rho}=1\right)$, charge and pairing correlations both decay as $1 / x^{2}$.

We calculated the correlation functions (5) with the CT-INT method, and plot the results in terms of the conformal distance [45] $\xi=L \sin (\pi r / L)$ to remove effects of the periodic boundary conditions. The results are shown in Fig. 4. For $\lambda<\lambda_{\mathrm{c}}=0.39(2)$, the numerical data are consistent with a power-law decay, as suggested by Eq. (8). Because charge correlations dominate over pairing correlations, we conclude that $K_{\rho}<1$. Moreover, Fig. 4 suggests that $K_{\rho}$ decreases from the noninteracting value $K_{\rho}=1$ with increasing $\lambda$, in accordance 

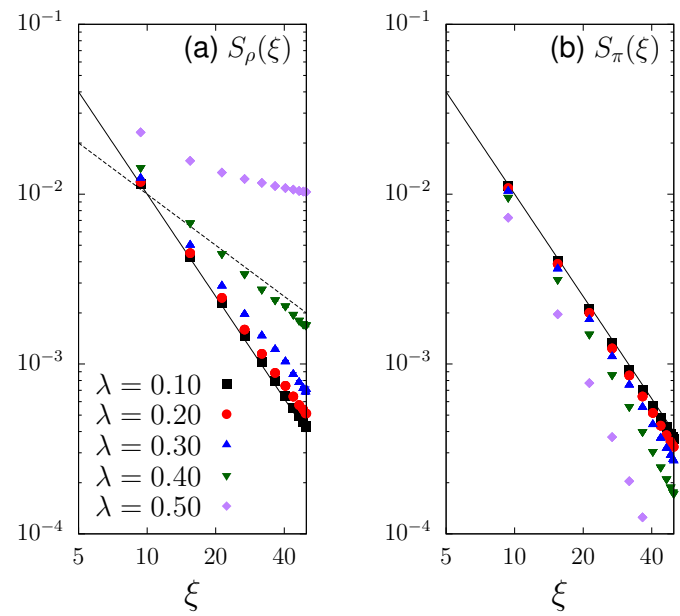

FIG. 4. (Color online) Charge and pairing correlation functions of the spinless Holstein model. Solid (dashed) lines illustrate $c / x^{2}(c / x)$. Here, $\omega_{0} / t=0.1, L=\beta t=50$. Results obtained with the CT-INT method.

with DMRG results [15]. The critical point is expected at $K_{\rho}=1 / 2$ (see discussion in Sec. VI), suggesting a $1 / x$ decay of charge correlations at $\lambda_{c}$ that is in satisfactory agreement with the data.

Figure 5 provides a more stringent test of the relationship between our numerical data and the bosonization results in Eq. (8). It shows results for the correlation functions for different system sizes $L$ at a given value of $\lambda$. If the correlations decay with a power-law determined by $K_{\rho}$, we expect data for different $L$ to fall onto the same straight line in a log-log plot (i.e., to have the same exponent). According to Fig. 5, this is indeed the case for $\lambda=0.2$. In contrast, for $\lambda=0.4$ and $\lambda=0.5$, corresponding to the Peierls phase, we find a violation of this "scaling". Instead of a power-law decay, the charge correlations exhibit long-range order, and the corresponding pairing correlations [Fig. 5(b3)] are consistent with an exponential decay at large distances.

\section{Spinful Holstein model}

For spinful fermions, the bosonization gives [44, 46]

$$
\begin{aligned}
& S_{\rho}(x)=-\frac{K_{\rho}}{\pi^{2} x^{2}}+\frac{B_{\rho}}{x^{K_{\rho}+K_{\sigma}}} \cos \left(2 k_{\mathrm{F}} x\right), \\
& S_{\pi}(x)=\frac{B_{\pi}}{x^{K_{\rho}^{-1}+K_{\sigma}}}, \\
& S_{\sigma}(x)=-\frac{K_{\sigma}}{4 \pi^{2} x^{2}}+\frac{B_{\sigma}}{x^{K_{\rho}+K_{\sigma}}} \cos \left(2 k_{\mathrm{F}} x\right)
\end{aligned}
$$

for a Luttinger liquid without a spin gap, and [44]

$$
\begin{aligned}
S_{\rho}(x) & =\frac{C_{\rho}}{x^{2}}+\frac{C_{\rho}^{\prime}}{x^{K_{\rho}}} \cos \left(2 k_{\mathrm{F}} x\right), \\
S_{\pi}(x) & =\frac{C_{\pi}}{x^{K_{\rho}^{-1}}}
\end{aligned}
$$
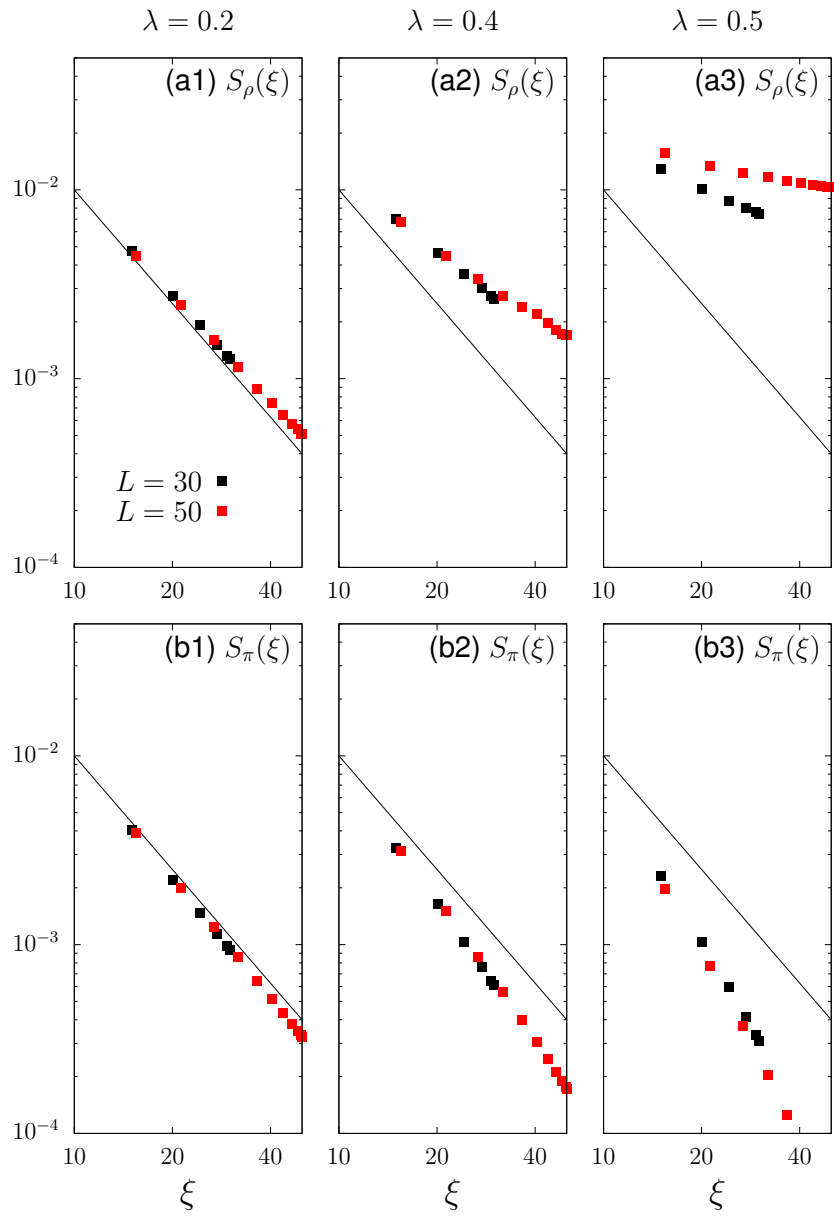

FIG. 5. (Color online) Charge and pairing correlations of the spinless Holstein model. Solid lines illustrate $c / x^{2}$. Here, $\omega_{0} / t=0.1, \beta t=L$. Results obtained with the CT-INT method.

for a Luther-Emery liquid with a gap for spin excitations. Given $\mathrm{SU}(2)$ spin symmetry, the value of $K_{\sigma}$ in Eq. (9) is fixed to 1, while the Luther-Emery correlation functions [Eq. (10)] are obtained by setting $K_{\sigma}=0$. The Peierls phase with a spin gap and long-range charge correlations formally corresponds to $K_{\rho}=K_{\sigma}=0$. In Eqs. (9) and (10), we included only the dominant $q=0$ part for the pairing correlators. Moreover, in Eq. (9), we neglected the $q=4 k_{\mathrm{F}}$ charge term because it is always subdominant for the cases considered here. Equations (9)(10) ignore possible logarithmic corrections, which can arise from marginally irrelevant operators [46, 47].

In both Luttinger liquids and Luther-Emery liquids, charge correlations dominate over pairing correlations for $K_{\rho}<1$. However, for Luttinger liquids $2 k_{\mathrm{F}}$ charge and spin correlations have exactly the same exponent [Eq. (9)]. This degeneracy can be lifted in favor of dominant charge correlations (and exponential spin correlations) by a spin gap [40]. As argued before [6, 18, 19], numerical results for the correlation functions of the spinful Holstein model are consistent with a spin gap. Figure 6 

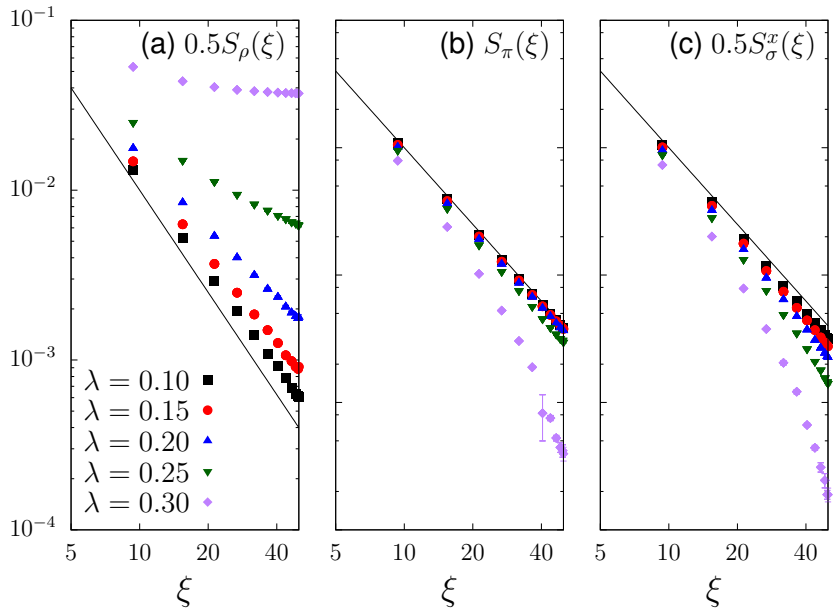

FIG. 6. (Color online) Charge, pairing, and spin correlation functions of the spinful Holstein model. The solid line illustrates $c / x^{2}$. Here, $\omega_{0} / t=0.5, \beta t=L=50$. Results obtained with the CT-INT method.

shows results for different values of $\lambda$. Starting with a $1 / x^{2}$ decay for $\lambda=0$, we observe an enhancement of charge correlations with increasing $\lambda$, and a suppression of both pairing and spin correlations. This behavior is captured by the Luther-Emery correlation functions in Eq. (10) with $K_{\rho}<1$, but not by the Luttinger liquid expressions of Eq. (9). The dominance of charge over pairing correlations $[6,18-21]$ contradicts earlier claims of a metallic phase with dominant superconducting correlations (i.e., $K_{\rho}>1$ ) [9], and the claim of $K_{\rho}=1$ in the metallic phase [10].

Figure 7 shows charge, pairing and spin correlation functions for different system sizes, with $\lambda$ fixed for each column. As for the spinless model, we can test if the data exhibit the scaling expected for power-law correlations. For $\lambda=0.1$, deep in the metallic phase, the results in Figs. 7(a1), (b1), and (c1) are consistent with a power-law decay. The absence of a clear exponential decay in Fig. 7(c1) suggests that the system sizes are not sufficient to reach the Luther-Emery fixed point where Eq. (10) holds. A clear exponential decay of spin correlations in the metallic phase has been observed in DMRG studies carried out at higher phonon frequencies where the metallic phase extends to larger values of $\lambda[20,21]$. The crossover from Luttinger liquid to Luther-Emery liquid behavior as a function of distance will be illustrated for the numerically more accessible attractive Hubbard model in Sec. VB.

For a stronger coupling $\lambda=0.2$ [Figs. 7(a2), (b2), (c2)], close to the critical point, we observe signatures of longrange charge order, and of a break-down of power-law scaling. Finally, for $\lambda=0.25$ [Figs. 7(a3), (b3), (c3)], corresponding to the Peierls phase, deviations from scaling are visible in all three channels. Charge correlations clearly reflect the long-range order, while pairing and spin correlations are consistent with an exponential decay.
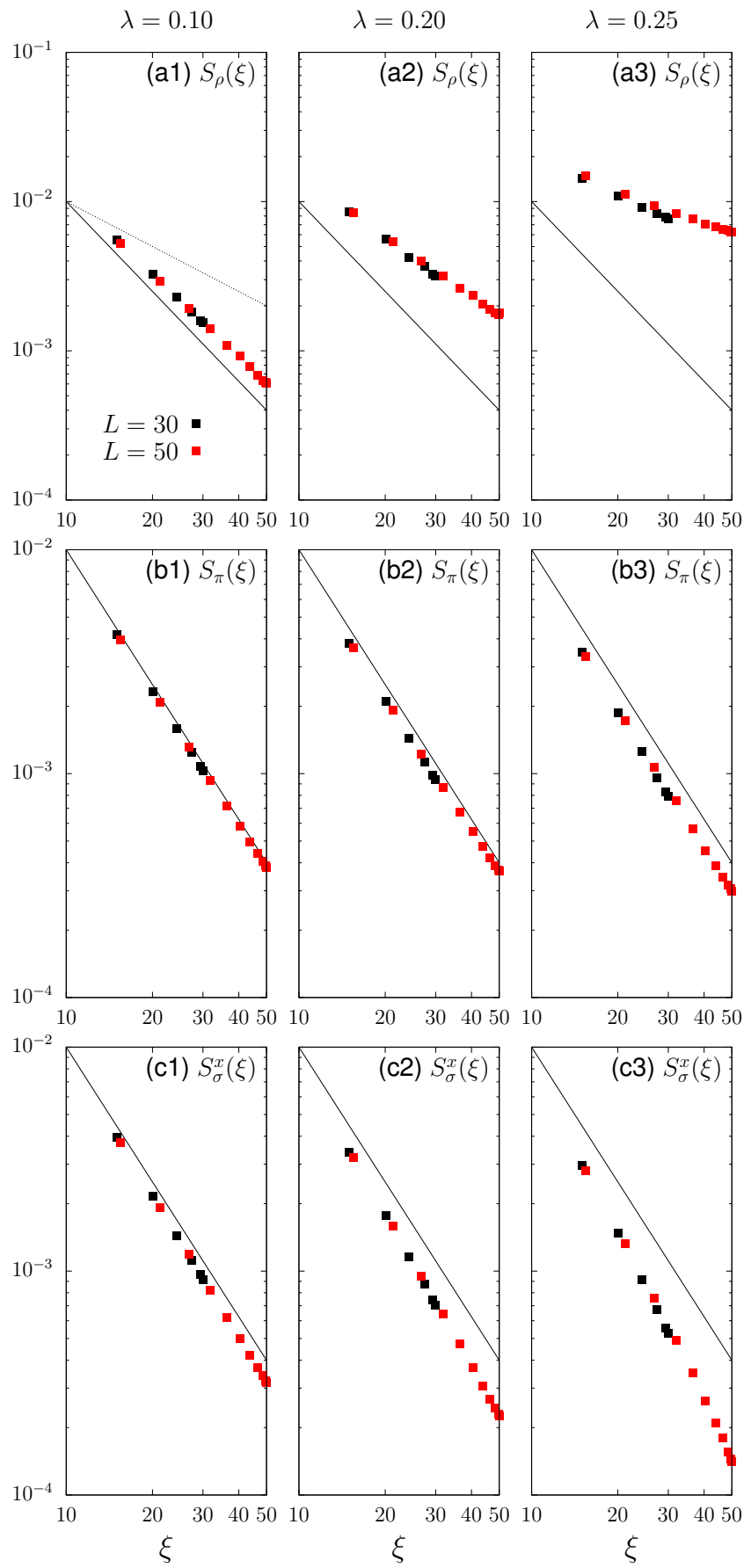

FIG. 7. (Color online) Charge, pairing, and spin correlation functions of the spinful Holstein model. Solid (dashed) lines illustrate $c / x^{2}(c / x)$. Here, $\omega_{0} / t=0.5, \beta t=L$. Results obtained with the CT-INT method.

\section{Luttinger liquid parameter $K_{\rho}$}

Our numerical results for the correlation functions may be explained using the bosonization expressions with suitable exponents. It is therefore of particular interest to calculate $K_{\rho}$ as a function of $\omega_{0}$ and $\lambda$. 
$K_{\rho}$ is routinely extracted from numerical results for the charge structure factor,

$$
K_{\rho}=s \pi \lim _{q \rightarrow 0} S_{\rho}(q) / q
$$

where $s=1(s=2)$ for spinful (spinless) models. The relation of $K_{\rho}$ to the $q=0$ charge fluctuations follows from $S_{\rho}(x)$ in Eqs. (8) and (9), the first term of which is directly proportional to $K_{\rho}$. Although $K_{\rho}$ is defined in the thermodynamic limit, a finite-size estimate can be obtained from

$$
K_{\rho}(L)=s \pi S_{\rho}\left(q_{1}\right) / q_{1},
$$

where $q_{1}=2 \pi / L$ is the smallest nonzero wavevector; finite-size scaling then gives, in principle, the physical value of $K_{\rho}$, although the scaling function is in general not known. The use of Eq. (12) is usually motivated by simplicity and the absence of (multiplicative) logarithmic corrections to the $q=0$ term in the correlation functions. Logarithmic corrections to the power-law decay of correlation functions may arise from marginally irrelevant operators [46-48], although we are not aware of explicit results for corrections to the $q=0$ term. Moreover, for the Luther-Emery fixed point, the identification of the constant $C_{\rho}$ with $K_{\rho}$ is expected to hold only for small spin gaps. We will demonstrate below that the spinful Holstein model, and also the attractive Hubbard model, are examples for which the determination of $K_{\rho}$ from Eq. (12) is problematic.

Using Eq. (12), $K_{\rho}$ for the spinful Holstein and the Holstein-Hubbard model has previously been extracted from QMC [9, 10] and DMRG [12] results. The large system sizes used (up to several hundred lattice sites) suggest the possibility of a reliable extrapolation to the infinite system. These works reported $K_{\rho}>1$ in the metallic phase $[9,12]$, thereby implying dominant pairing correlations. Later, it was argued that $K_{\rho}>1$ due to logarithmic corrections and that the true value is $K_{\rho}=1$ in the metallic phase [10], as in the attractive Hubbard model. However, the observation of dominant charge correlations (see above and Refs. 6, 18-21) in the metallic phase seems compatible only with $K_{\rho}<1$.

We used the CT-INT method to calculate $K_{\rho}(L)$ via Eq. (12) for the spinful Holstein model. The results for $\omega_{0} / t=0.5$ as a function of $\lambda$ are shown Fig. 8. In agreement with previous work, we find $K_{\rho}(L)>1$ for $\lambda>0$ in the metallic phase for the spinful Holstein model, see Fig. 8(a). Starting from $\lambda=0, K_{\rho}(L)$ initially increases with increasing $\lambda$, before it starts to decrease in the vicinity of the critical value $\lambda_{\mathrm{c}}=0.23(1)$. A very similar behavior was observed before [10]. Crucially, $K_{\rho}(L)$ takes on even larger values if we increase the phonon frequency from $\omega_{0} / t=0.5$ to $\omega_{0} / t=5$; for the latter, the metallic phase extends up to $\lambda_{\mathrm{c}} \approx 0.5[10,11]$. This observation seems to rule out retardation effects as the reason for the slow convergence of $K_{\rho}$ [18].

Further insight can be gained from the spinless Holstein model. In Fig. 8(b), we see that $K_{\rho}(L) \lesssim 1$ even
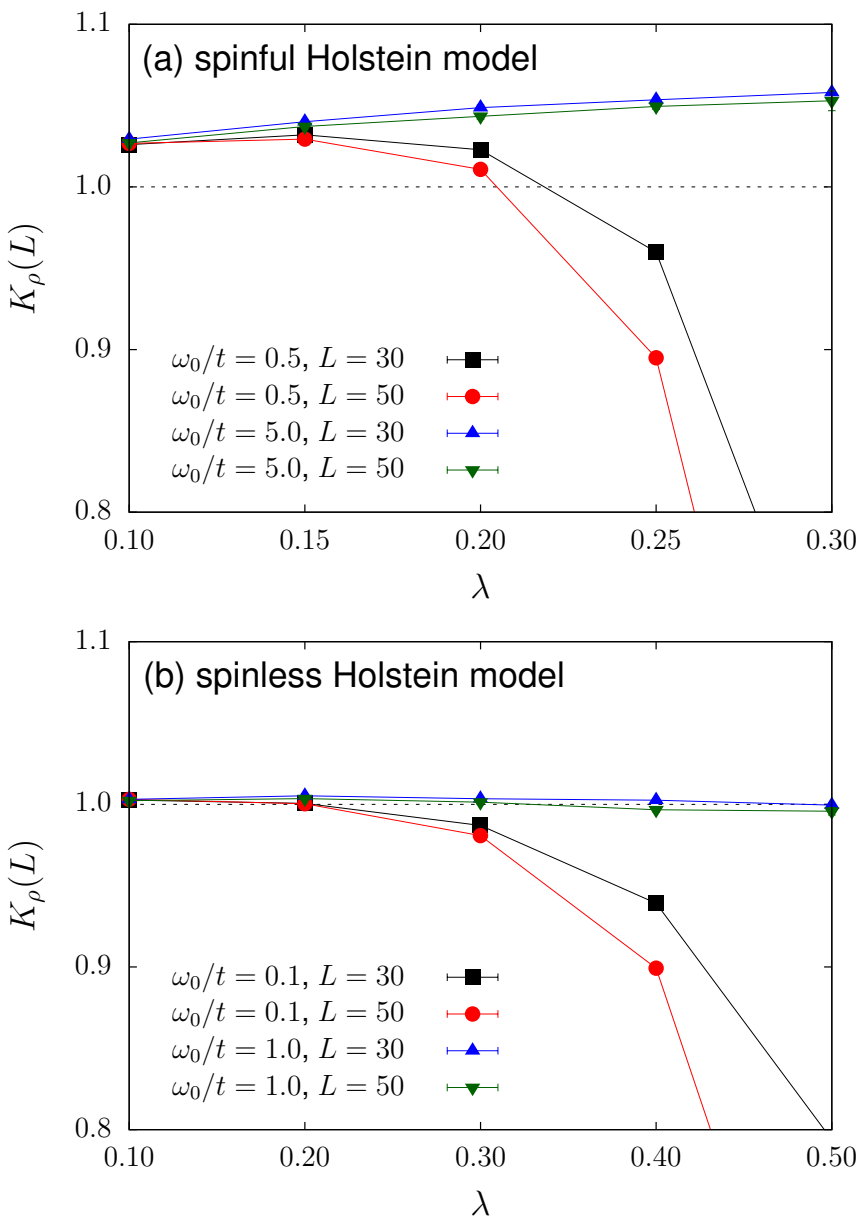

FIG. 8. (Color online) $K_{\rho}(L)$ [Eq. (12)] for (a) the spinful and (b) the spinless Holstein model. Here, $\beta t=L$. Results obtained with the CT-INT method.

for the rather small lattice sizes accessible with the CTINT method. In contrast to the spinful model, the finitesize extrapolated values of $K_{\rho}$ are always smaller than 1 for nonzero $\lambda[15]$, consistent with the dominant charge correlations in Fig. 4. Moreover, the DMRG was able to show that $K_{\rho}=1 / 2$ coincides within the numerical accuracy with the critical point of the Peierls transition, as expected from bosonization results for the related $t-V$ model of spinless fermions [15].

By combining the above results, we arrive at the following conclusions. For the spinful Holstein model, the use of Eq. (12) gives results for $K_{\rho}$ that are inconsistent with the correlation functions, which show a generic dominance of charge over pairing correlations. Even in the absence of accurate estimates of $K_{\rho}$, the behavior of the correlation functions hence establishes the repulsive nature of the Luther-Emery phase. In contrast, for the spinless Holstein model, the values of $K_{\rho}$ determined by large-scale DMRG calculations [15] are compatible [49] with the behavior of correlation functions. Since the retardation of the phonon-mediated interaction is identical for the spinful and the spinless model for the same $\omega_{0} / t$ 


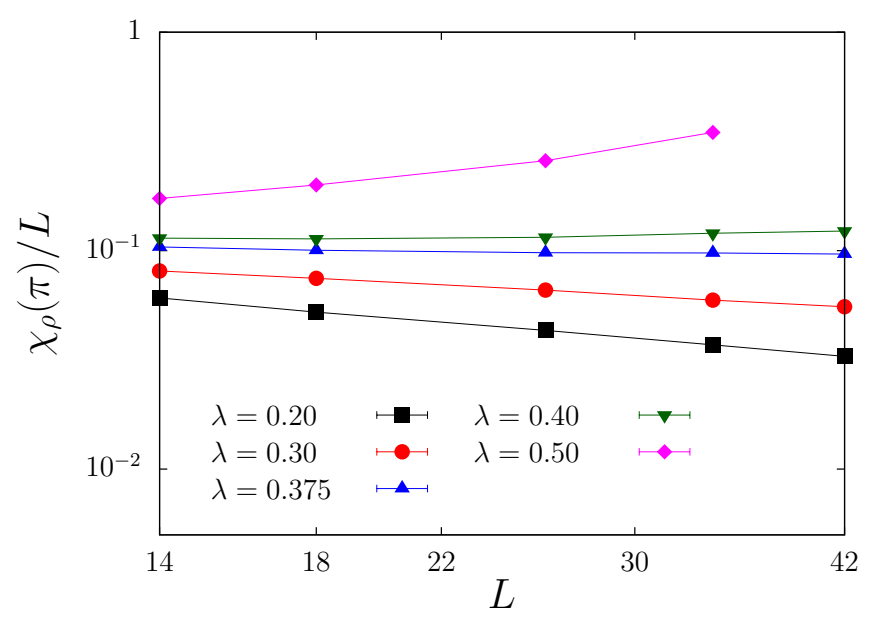

FIG. 9. (Color online) Finite-size scaling of the charge susceptibility $\chi_{\rho}(\pi)$ [Eq. (13)] for the spinless Holstein model. Here, $\omega_{0} / t=0.1, \beta t=L$. Results obtained with the CT-INT method.

[ $\omega_{0} / t$ is actually smaller in Fig. 8(b) than in Fig. 8(a)], it cannot explain $K_{\rho}>1$ in the spinful case. On the other hand, the key difference is the additional energy scale of the spin gap arising from attractive backscattering in the spinful case. We will return to this point in Sec. V.

\section{Charge susceptibility}

While the onset of long-range order can be tracked using a finite-size scaling of charge correlations, as shown in Fig. 3, previous QMC studies of the phase diagram mainly relied on the charge susceptibility

$$
\chi_{\rho}(Q)=\sum_{r=1}^{L} e^{i Q r} \int_{0}^{\beta} d \tau\left\langle\hat{n}_{r}(\tau) \hat{n}_{0}(0)\right\rangle
$$

at the ordering wavevector $Q=2 k_{\mathrm{F}}=\pi$. In principle, the susceptibility has a more favorable scaling (a faster divergence in the ordered phase) with system size [50, 51]. However, because $\chi_{\rho}$ involves the charge correlation function, it is also affected by the spin gap. We therefore discuss the expected and the observed behavior of the charge susceptibility for Holstein models. To compare to existing work, we consider a phonon frequency $\omega_{0} / t=$ 0.1 in the spinless case, and $\omega_{0} / t=0.5$ in the spinful case. Results for the $t-V$ and the $U-V$ extended Hubbard model will be shown in Sec. V.

As shown in the appendix, assuming a power-law decay of $2 k_{\mathrm{F}}$ charge correlations of the form $(-1)^{r} r^{-\alpha}$, the susceptibility scales as

$$
\chi_{\rho}(\pi) / L \sim C L^{1-\alpha} .
$$

Let us analyze the behavior of $\chi_{\rho}(\pi) / L$ for Luttinger and Luther-Emery liquids, focusing on $K_{\rho} \leq 1$.
For a spinless Luttinger liquid, such as the spinless Holstein model for $\lambda<\lambda_{c}$, we have $\alpha=2 K_{\rho}$ [see Eq. (8)]. At half-filling, umklapp scattering is irrelevant for $K_{\rho} \geq 1 / 2$, and relevant for $K_{\rho}<1 / 2$. Equation (14) suggests the following behavior: for $K_{\rho}=1, \chi_{\rho}(\pi) / L \sim \ln L / L$ and hence $\chi_{\rho}(\pi) / L \rightarrow 0$ for $L \rightarrow \infty$. Similarly, $\chi_{\rho}(\pi) / L \rightarrow 0$ for $1 / 2<K_{\rho}<1$. At the critical value $K_{\rho}=1 / 2$, we have $\chi_{\rho}(\pi) / L \rightarrow C$. For $K_{\rho}<1 / 2, \chi_{\rho}(\pi) / L$ diverges as a function of $L$. In particular, $\chi_{\rho}(\pi) / L \sim L$ for $K_{\rho}=0$ (corresponding to long-range charge order). Hence, we should see a crossover in the behavior of $\chi_{\rho}(\pi) / L$ as a function of $K_{\rho}$ (or, equivalently, the interaction strength), and can identify the critical point from the onset of diverging behavior. The above considerations are borne out by the numerical results for the spinless Holstein model (2) shown in Fig. 9. Despite the limited system sizes, the critical value $\lambda_{\mathrm{c}} \approx 0.40$ agrees well with large-scale DMRG results $[14,15]$.

For a spinful Luttinger liquid $\alpha=K_{\rho}+K_{\sigma}$ [Eq. (9)]. If $K_{\sigma}=1$ by symmetry, then $\chi_{\rho}(\pi) / L \sim L^{-K_{\rho}}$. Therefore, we expect $\chi_{\rho}(\pi) / L \rightarrow 0$ for $0<K_{\rho}<1$ (the range where correlations decay with a power-law), and a divergence of $\chi_{\rho}(\pi) / L$ in the ordered state where the correlations approach a finite value at large distances.

Finally, for a Luther-Emery liquid, as realized in the metallic phase of the spinful Holstein model, $2 k_{\mathrm{F}}$ charge correlations decay with $\alpha=K_{\rho}$ [Eq. (10]. Consequently, Eq. (14) gives $\chi_{\rho}(\pi) / L \rightarrow C$ for $K_{\rho}=1$, but $\chi_{\rho}(\pi) / L \sim$ $L^{\epsilon}$ with $\epsilon=1-\alpha>0$ and hence $\chi_{\rho}(\pi) / L \rightarrow \infty$ for any $K_{\rho}<1$. Therefore, a metallic phase with $K_{\rho}<1$ (dominant charge correlations, as observed for the Holstein model) cannot be distinguished from a long-rangeordered Peierls phase from the qualitative behavior of $\chi_{\rho}(\pi) / L$ alone. This complication appears to have been overlooked in Refs. 9, 10, and 27 where $\chi_{\rho}(\pi)$ was used to track the Peierls transition in spinful models. The scaling $\chi_{\rho}(\pi) \sim L^{2-K_{\rho}}$ for a Luther-Emery liquid was given in Ref. 52. In principle, the Luther-Emery and CDW phases may be distinguished by the different divergences of $\chi_{\rho}(\pi) / L$. Given reliable estimates of $K_{\rho}$, the LutherEmery phase can be identified by plotting $\chi_{\rho}(\pi) L^{1-K_{\rho}}$, which approaches a constant at large $L$ [52].

Let us compare these predictions to numerical data for the spinful Holstein model. The crossover from Luttinger liquid to Luther-Emery liquid behavior in finite systems corresponds to a change of the exponent $\alpha$ from $K_{\rho}+K_{\sigma}$ to $K_{\rho}$. In the case of the Holstein model, the charge correlation functions in Fig. 7(a) reveal an exponent $1<$ $\alpha<2$ (the solid line indicates $\alpha=2$, the dashed line $\alpha=1$ ) even though the dominance of charge over pairing correlations implies $K_{\rho}<1$. This suggests that on the length scales accessible in our results, $K_{\sigma}$ has not scaled to zero, putting us between the two fixed points.

The problems in resolving the spin gap and the correct long-distance behavior of the correlation functions also affect the charge susceptibility. In contrast to the analytical predictions of a divergent $\chi_{\rho}(\pi) / L$ for any $K_{\rho}<1$ in a Luther-Emery liquid, the data for $\chi_{\rho}(\pi)$ in Fig. 10(a) 

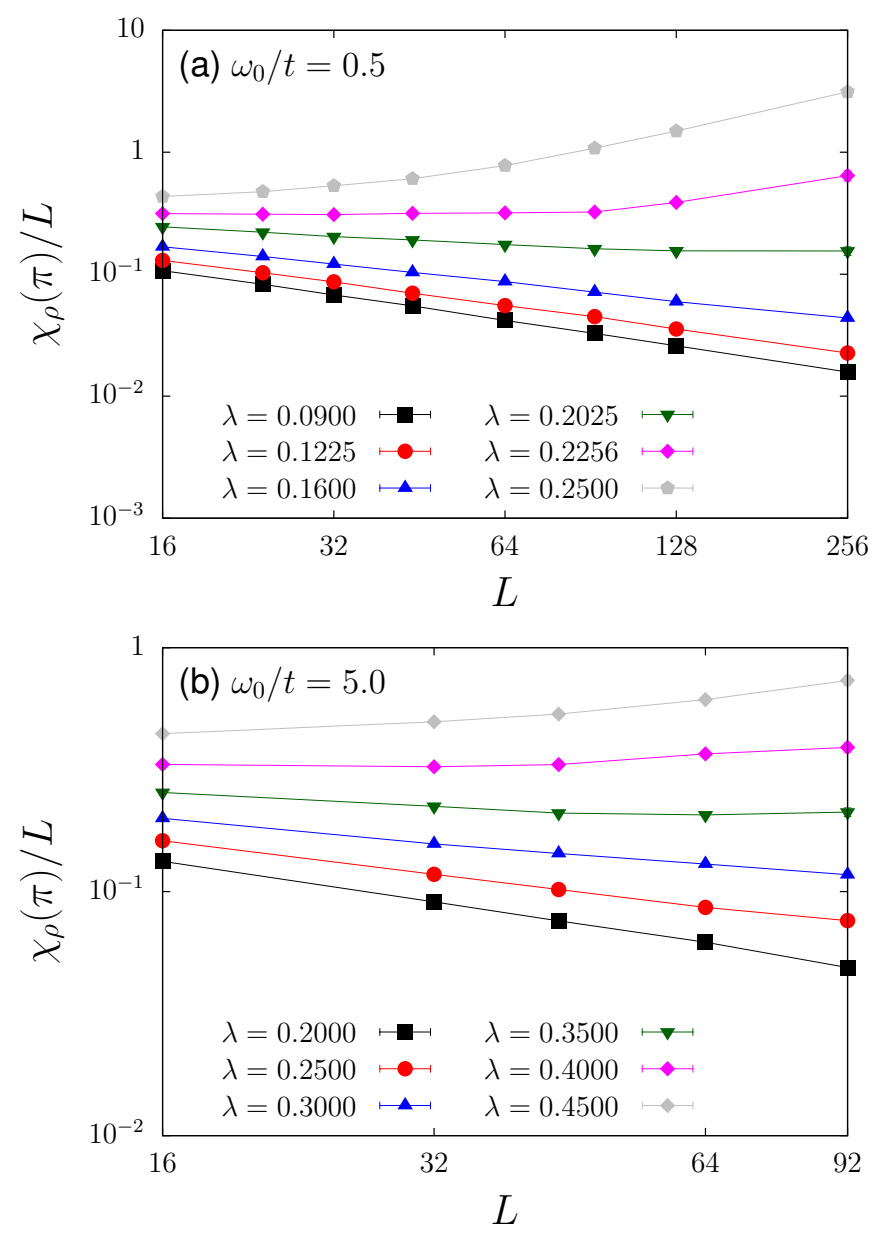

FIG. 10. (Color online) Finite-size scaling of the charge susceptibility $\chi_{\rho}(\pi)$ for the spinful Holstein model for (a) $\omega_{0} / t=0.5$, and (b) $\omega_{0} / t=5$. Here, $\beta t=L$. Results obtained in the SSE representation.

strongly resemble the spinless case shown in Fig. 9. For small values of $\lambda, \chi_{\rho}(\pi) / L \rightarrow 0$, whereas for sufficiently large $\lambda$ it diverges. This behavior is consistent with previous work [9, 10, 27], cf. Fig. 3(a) in Ref. 10.

Based on the incorrect assumption of $\chi_{\rho}(\pi) / L \rightarrow 0$ in the metallic phase, the phase boundary for the Peierls transition was determined in Ref. 10. Similarly, from Fig. 10(a), we may (incorrectly) estimate $\lambda_{c} \approx 0.23$, in accordance with Ref. 10. The DMRG estimate (from the opening of the two-particle gap) is $\lambda_{c} \approx 0.25$ [11]. For $\omega_{0} / t=1$, the transition was reported to occur at $\lambda_{c} \approx 0.25$ based on $\chi_{\rho}(\pi) / L[10]$, whereas a rough DMRG estimate (from the order parameter) is $\lambda_{c} \approx 0.3$ [39]. The spurious crossover in the behavior of $\chi_{\rho}(\pi) / L$ is also apparent in Fig. 10(b) for $\omega_{0} / t=5$. Similar data were used before to estimate $\lambda \approx 0.5$ [10]. A comparison of QMC and DMRG results can be made for the HolsteinHubbard model with $\omega_{0} / t=5$ and $U / t=1$. The QMC critical value is $\lambda_{c} \approx 0.65$ [10], whereas DMRG estimates are $\lambda_{c} \approx 0.8-1.0[11]$ and $\lambda_{c} \approx 0.75$ [12].
Our discussion reveals that the Peierls critical point for the spinful Holstein model (or other models exhibiting a transition from a Luther-Emery to a CDW phase) cannot be determined from a qualitative analysis (divergent or not) of $\chi_{\rho}(\pi) / L$ because the latter diverges in both phases on sufficiently large systems. Even a quantitative analysis of the divergent behavior will be affected by the spin gap. Since the latter partially suppresses the divergence of $\chi_{\rho}(\pi) / L$ for small $L$, critical values obtained from the susceptibility are expected to be larger than the true values. Given the importance of the HolsteinHubbard model for our understanding of electron-phonon physics, it is highly desirable to use alternative methods to improve the accuracy of the phase diagram.

\section{FERMIONIC MODELS}

In this section, we consider purely electronic models of spinful and spinless fermions that exhibit a transition from a metallic phase to a CDW insulator. This allows us to separate retardation effects from those of backscattering (leading to a spin gap) and umklapp scattering (leading to a charge gap). The availability of exact analytical results provides a stringent test for the numerical methods also used to study electron-phonon models. After demonstrating that the spinless fermion problem is well accessible numerically, leading to a very good agreement with analytical results, we will show that the extended Hubbard model exhibits many of the issues encountered in numerical simulations of the spinful Holstein model. Because of the better scaling with system size, all results of this section were obtained in the SSE representation.

\section{A. Spinless $t-V$ model}

The model of spinless fermions defined by Eq. (4) captures the Luttinger liquid to CDW insulator transition also observed for the spinless Holstein model. Importantly, it does not involve any retardation effects, and can be solved exactly by the Bethe ansatz [53], thereby providing full knowledge of the correlation functions and the phase diagram (see Ref. 54 and references therein).

Figure 11 shows $K_{\rho}(L)$ as a function of inverse system size. In the metallic phase, which exists for $V / t<2$, the numerical values extrapolate to the exact values [55]

$$
K_{\rho}=\frac{\pi}{2} \frac{1}{\arccos \left(-\frac{V}{2 t}\right)} .
$$

Close to the transition, the extrapolation becomes more difficult. In particular, $K_{\rho}$ should take on the value 0.5 exactly at the critical point $V / t=2$, and zero for $V / t>$ 2. Similar results were obtained in a previous DMRG study [56], as well as for the spinless Holstein model [15].

The charge correlation function $S_{\rho}(\xi)$ is shown for different values of $V / t$ in Fig. 12. In the metallic phase 


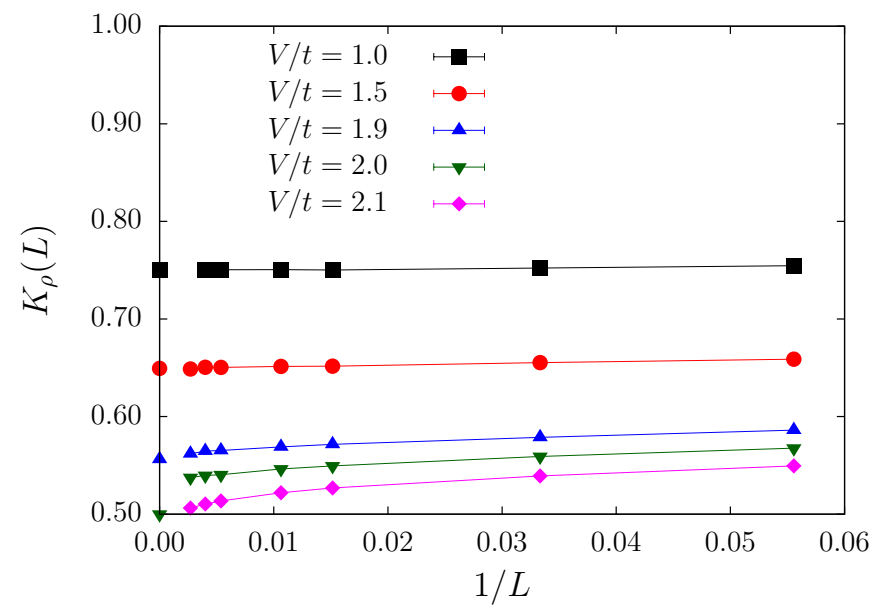

FIG. 11. (Color online) (a) $K_{\rho}(L)$ [Eq. (12)] for the spinless $t$ $V$ model. Here, $\beta t=2 L$. The values for $L=\infty$ were obtained from Eq. (15). Results obtained in the SSE representation.
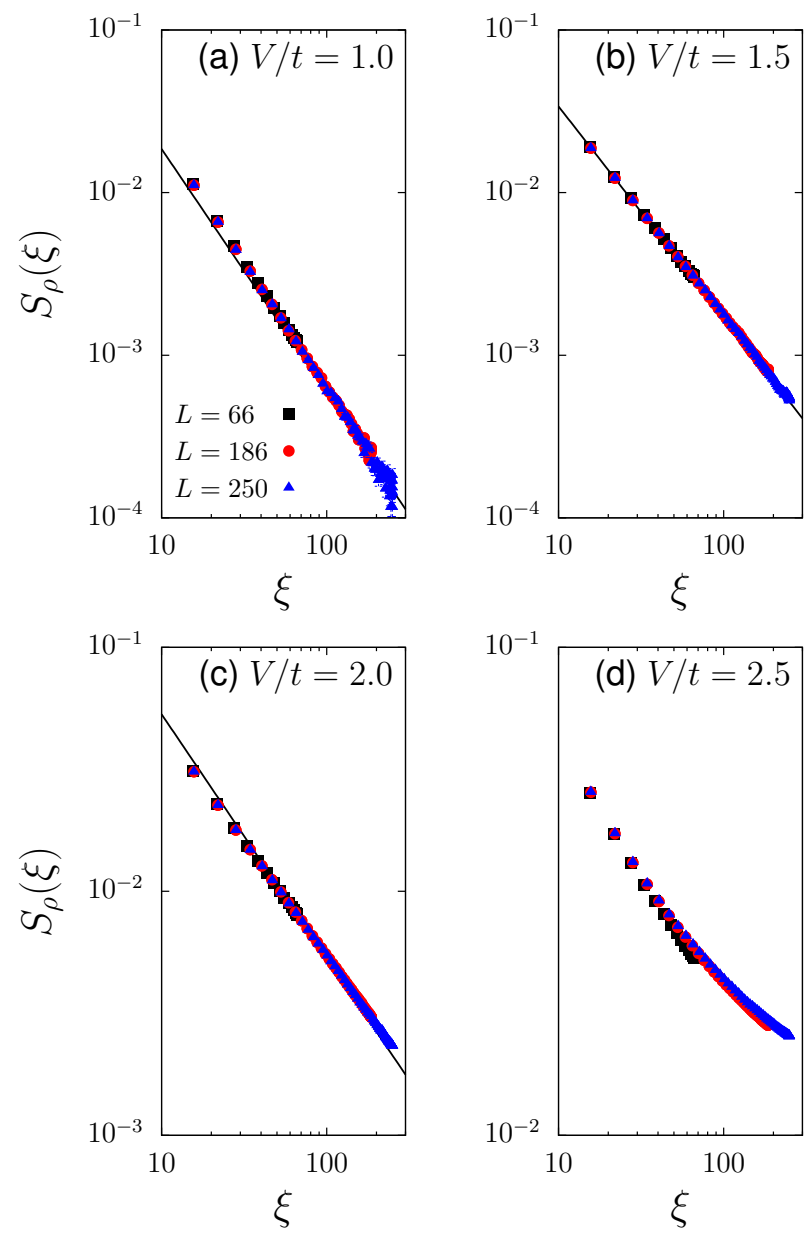

FIG. 12. (Color online) Charge correlation functions for the spinless $t$ - $V$ model. Solid lines correspond to fits to $a / x^{2 K_{\rho}}$ using results for $r>10$ and $L=250$, with $K_{\rho}$ from Eq. (15). Here, $\beta t=2 L$. Results obtained in the SSE representation.
$(V / t<2)$, we see a power-law decay for all system sizes considered. Apart from the expected deviations at very small distances, the data match the form $x^{-2 K_{\rho}}$ [Eq. (8)] with $K_{\rho}$ taken from Eq. (15). Even exactly at the critical point $V / t=2$, the numerical results do not indicate logarithmic corrections on the length scales considered. For $V / t=2.5$, we see long-range order. Similar to the Holstein models, we have a collapse of results for different $L$ in the metallic phase [Figs. 12(a)-(c)], but not in the insulating phase [Fig. 12(d)].

The charge susceptibility $\chi_{\rho}(\pi)$ is shown in Fig. 13(a). Since $S_{\rho}(\xi)$ behaves as expected from the bosonization, the numerical results for $\chi_{\rho}(\pi) / L$ are consistent with the discussion in Sec. IV D. For $V / t<2, \chi_{\rho}(\pi) / L$ goes to zero with increasing system size, whereas it diverges for $V / t>2$. The susceptibility can therefore be used to determine the critical value of the transition even on moderately large systems. The same conclusion holds for the spinless Holstein model.

Finally, we turn to the charge order parameter as an alternative way to track the phase transition. To this end, we consider the charge correlations at the largest distance $L / 2$ for different system sizes $L$, shown in Fig. 13(b). In the metallic phase, this quantity is expected to be zero in the thermodynamic limit, and nonzero in the chargeordered insulating phase. The data in Fig. 13(b) show that the scaling of the charge correlations allows to quite accurately determine the critical value of the phase transition. The same is true for the extended Hubbard model (not shown), and we therefore expect this order parameter to be a reliable tool to determine the transition for electron-phonon models.

\section{B. Attractive Hubbard model}

In Sec. IV we identified the existence of a (small) spin gap in the spinful Holstein model as a significant complication in finite-size studies. Because the retarded interaction and the nonintegrability of the Holstein model make further progress difficult, we turn to the exactly solvable attractive Hubbard model [Eq. (3) with $V=0$ ].

At half-filling, the attractive Hubbard model is related by a canonical transformation to the repulsive Hubbard model. From bosonization/RG studies [57], it is known that its ground state is metallic for any $U<0$ and has a gap for spin excitations. It therefore provides a numerically accessible realization of the Luther-Emery fixed point. The absence of phonons permits significantly larger system sizes to be simulated, and eliminates any uncertainties from the bosonization/RG treatment related to a phonon energy scale.

The exact relation to the repulsive Hubbard model has important consequences. The value of $K_{\rho}$ is fixed to 1 for any $U<0$ because $K_{\rho}$ in the attractive model corresponds to $K_{\sigma}$ in the repulsive model [where $K_{\sigma}=1$ because of SU(2) spin symmetry]. Additionally, $K_{\sigma}=0$ as a result of the spin gap. A value $K_{\rho}=1$ implies that 

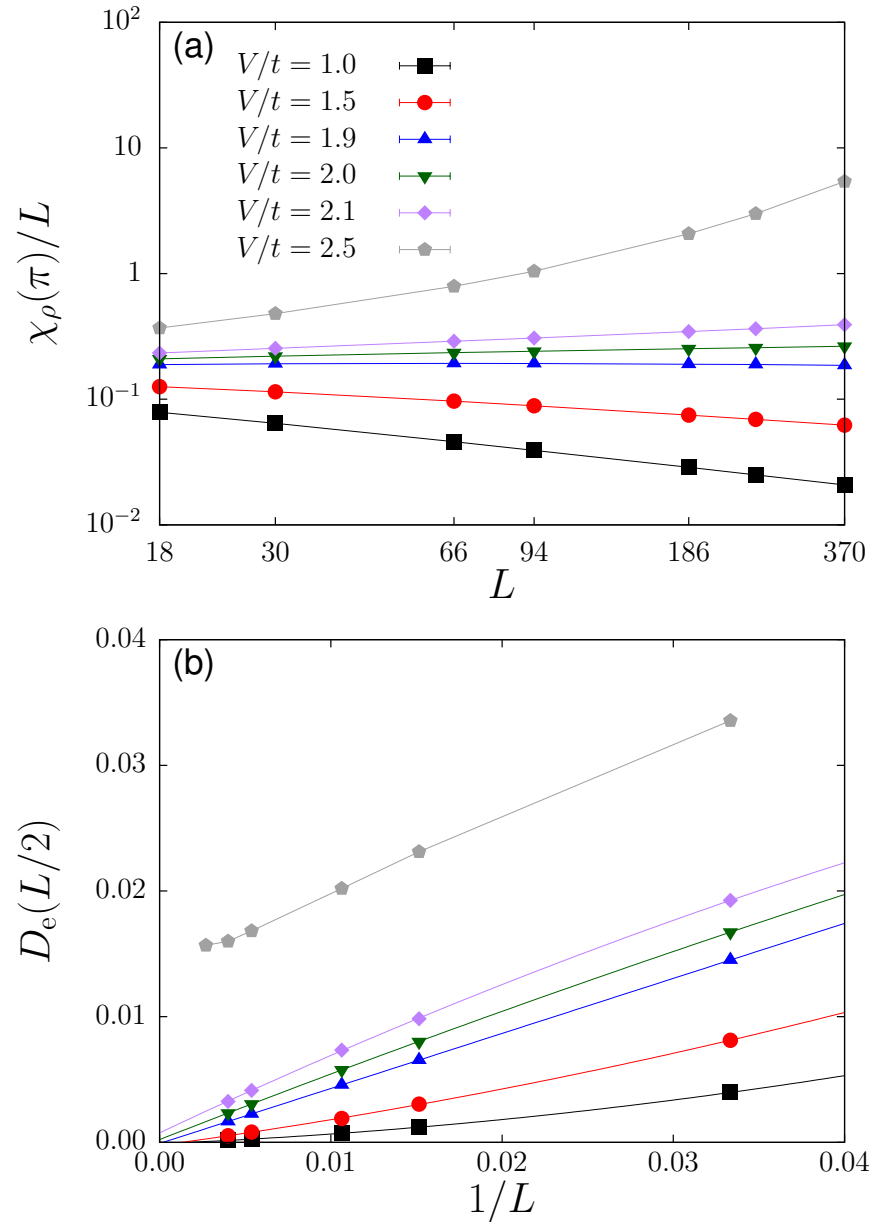

FIG. 13. (Color online) Finite-size scaling of (a) the charge susceptibility and (b) the order parameter for the spinless $t-V$ model. Here, $\beta t=2 L$. Results obtained in the SSE representation.

charge and pairing correlation functions remain degenerate for any $U$ [cf. Eq. (10)], and their $2 k_{\mathrm{F}}$ components are expected to decay as $x^{-1} \sqrt{\log (x)}[58,59]$.

Figure 14(a) shows $K_{\rho}(L)$ from simulations in the SSE representation on up to $L=370$ sites. In agreement with previous findings [10], $K_{\rho}(L)$ is significantly larger than 1 , and for a given $L$ increases with increasing $U$, reminiscent of the increase of $K_{\rho}(L)$ with increasing $\lambda$ in Fig. 8(a). The convergence with system size is very slow, making it challenging to obtain $K_{\rho}=1$ from an unbiased extrapolation. The relation between $K_{\rho}$ of the attractive and $K_{\sigma}$ of the repulsive Hubbard model is reflected in the observation that $K_{\sigma}(L)>1$, with a very slow convergence to the value $K_{\sigma}=1$ implied by symmetry (data not shown). A similarly slow convergence has also been observed for the repulsive Hubbard model with open boundaries, where $K_{\sigma}(L)$ was determined from fits to the local density of states [60]. In the attractive case considered here, Fig. 14(b) shows that $K_{\sigma}(L)<1$, and that the numerical data are consistent with $K_{\sigma}=0$.
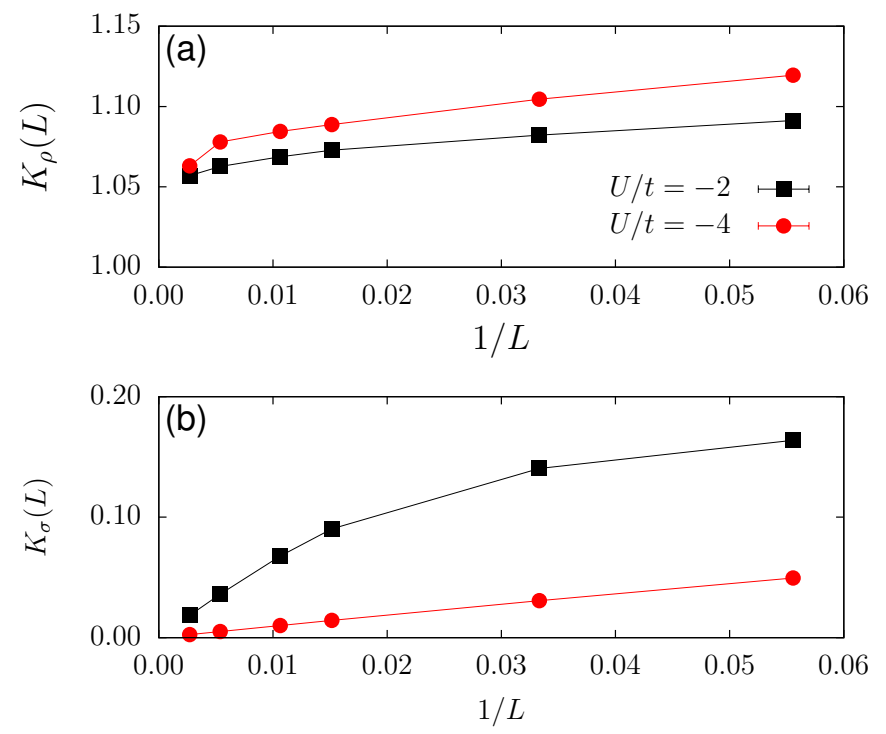

FIG. 14. (Color online) (a) $K_{\rho}(L)$ and (b) $K_{\sigma}(L)=$ $2 \pi S_{\sigma}\left(q_{1}\right) / q_{1}$ for the attractive Hubbard model. Here, $\beta t=$ $2 L$. Results obtained in the SSE representation.

Similar results have been obtained before for the Holstein model [6]. Values $K_{\sigma}(L)<1$ in numerical simulations are a reliable indicator for the existence of a spin gap [61].

Given the slow convergence of $K_{\rho}(L)$ for the attractive Hubbard model, the reliability of estimates of $K_{\rho}$ for the spinful Holstein model has to be questioned. As mentioned above, the slow convergence may be attributed to unknown logarithmic corrections [46-48]. While such corrections arise in the repulsive Hubbard model from the marginally irrelevant backscattering term, the umklapp term is marginally relevant (since $K_{\rho}=1$ by symmetry) in the attractive model. Logarithmic corrections are typically absent in spinless models, in accordance with our findings. The deviations of $K_{\rho}(L)$ from the expected value $K_{\rho}=1$ for a given $L$ are more pronounced for stronger interactions (larger spin gaps), similar to Ref. 60. Finally, in a Luther-Emery phase, the factor $C_{\rho}$ in the first term in $S_{\rho}$ in Eq. (10) may not be identical to $K_{\rho}$, and logarithmic corrections may arise.

Given a certain spin gap as a result of attractive backscattering, we expect to see physics reminiscent of the Tomonaga-Luttinger fixed point for distances small compared to the inverse of the spin gap, and LutherEmery behavior at large distances. The significantly larger system sizes accessible for the attractive Hubbard model permit us to illustrate this fixed-point crossover by comparing the numerical correlation functions to the Luttinger liquid and Luther-Emery results given in Eqs. (9) and (10), respectively.

For $U / t=-1$, the charge correlations shown in Fig. 15(a) decay with an exponent close to 2 at small distances, as expected from Eq. (9) for $K_{\sigma}=1, K_{\rho}=1$. With increasing distance $\xi$, we observe a crossover of the exponent caused by $K_{\sigma} \rightarrow 0$. For $U / t=-2$, the spin 

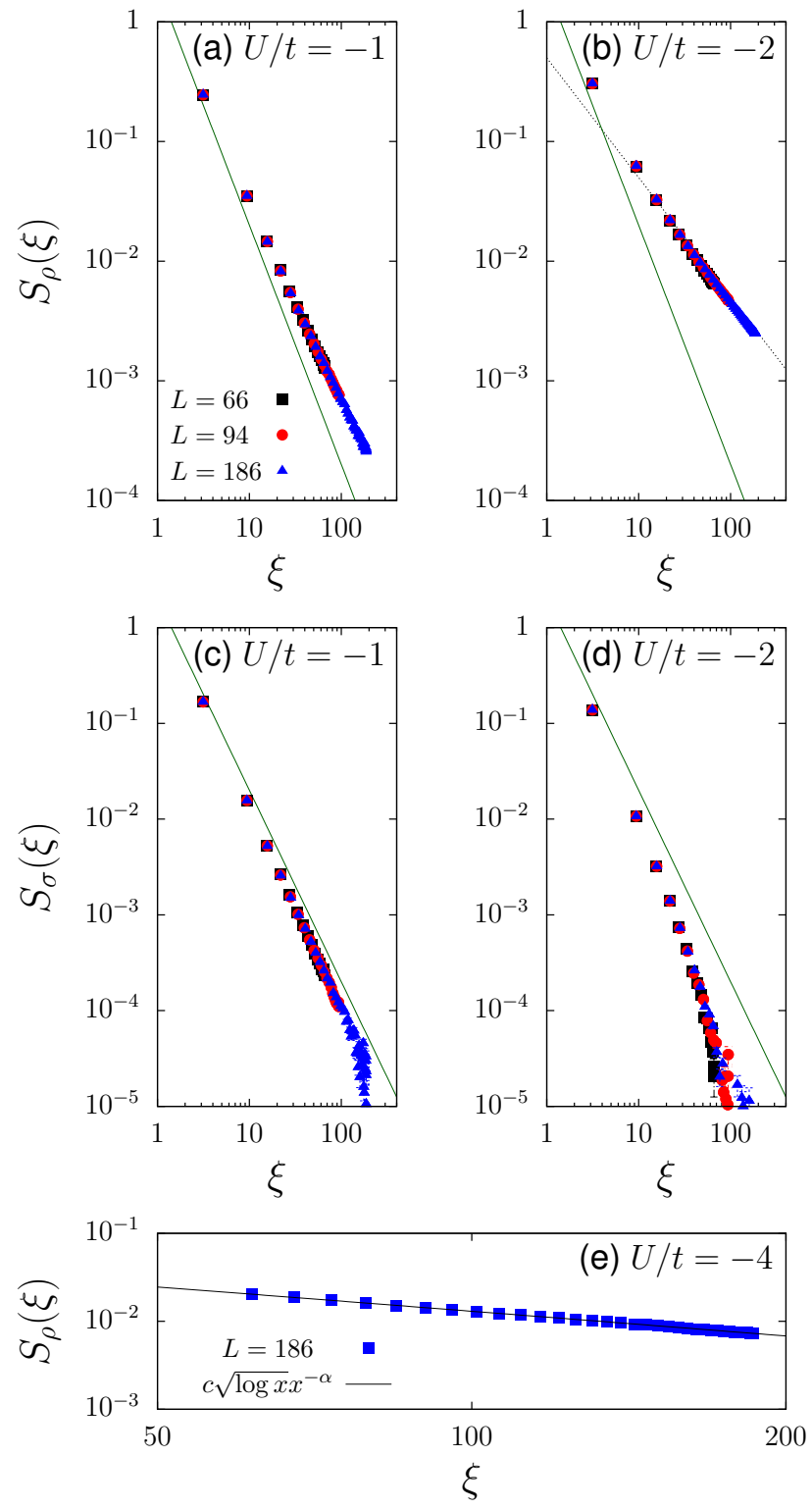

FIG. 15. (Color online) Charge and spin correlation functions for the attractive Hubbard model. In (a) - (d), solid (dashed) lines illustrate $c / x^{2}(c / x)$. Here, $\beta t=2 L$. Results obtained in the SSE representation.

gap is significantly larger, and the crossover from $x^{-2}$ to approximately $x^{-1}$ is visible in Fig. 15(b). Regardless of the crossover, we observe a collapse of data for different system sizes over the whole range of $\xi$. The spin correlations for $U / t=-1$, shown in Fig. 15(c), decay almost as in a gapless system (i.e., as $x^{-2}$ ), whereas for $U / t=-2$ the results are compatible with an exponential decay [Fig. 15(d)]. Hence, on length scales smaller than the inverse spin gap, charge correlations decay faster than in the thermodynamic limit, whereas spin correlations decay slower. While such a crossover is also expected for the Holstein model, the range of numerically accessible system sizes is insufficient to observe it numerically.

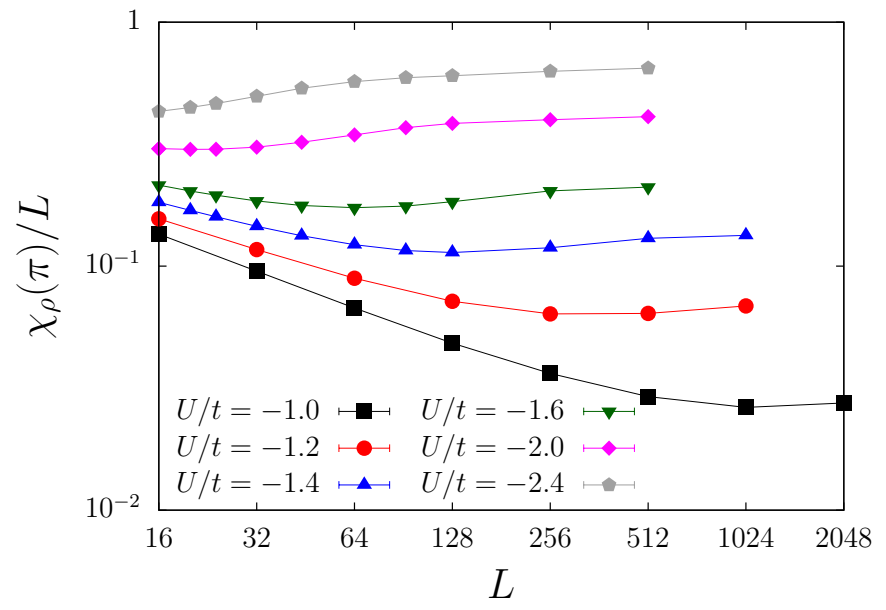

FIG. 16. (Color online) Finite-size scaling of the charge susceptibility for the attractive Hubbard model. Here, $\beta t=2 L$. Results obtained in the SSE representation.

Figure 15(e) shows the charge correlations for $U / t=$ -4 , where the spin gap is large enough to be resolved even on intermediate system sizes. Also shown is a fit to the prediction $c \sqrt{\log x} x^{-\alpha}$ from the bosonization (solid line) using data for $L=186$ and $r \geq 20$. The fit is in satisfactory agreement with the data; we find an exponent $\alpha \approx 1.03$ compatible with Fig. 14 and indicative of possible logarithmic corrections [46-48, 60] in addition to the crossover due to the spin gap.

The crossover in the correlation functions also affects the scaling of the charge susceptibility. If $S_{\rho}(x)$ decays faster than $x^{-1}, \chi_{\rho}(\pi) / L \rightarrow 0$ for $L \rightarrow \infty$. This is well visible in Fig. 16 for small $U / t$. For example, for $U / t=-1, \chi_{\rho}(\pi) / L$ decreases with increasing $L$ up to $L=1024$, well beyond the system sizes accessible for electron-phonon models. If the decay is exactly $x^{-1}$, $\chi_{\rho}(\pi) / L$ should approach a constant at large $L$. However, the logarithmic correction $\sqrt{\log x}$ for the attractive Hubbard model gives rise to a slow divergence of $\chi_{\rho}(\pi) / L$ with system size, as visible in Fig. 16 at large $L$. The different behavior of $\chi_{\rho}(\pi) / L \rightarrow 0$ for different $U / t$ in Fig. 16 may be mistaken as evidence for a transition to a CDW-ordered phase, even though the model remains metallic. Similar deviations from the expected behavior of $\chi_{\rho}(\pi) / L$ on finite systems can be observed for the spinful Holstein model (see Fig. 10).

\section{Extended Hubbard model}

Whereas the attractive Hubbard model is always a Luther-Emery metal, we can study the transition to a charge-ordered insulating phase by adding a repulsive nearest-neighbor interaction $V \sum_{i} \hat{n}_{i} \hat{n}_{i+1}$, see Eq. (3). This extended Hubbard model captures the existence of a spin gap in the metallic phase and $2 k_{\mathrm{F}} \mathrm{CDW}$ order in the insulating phase, similar to the Holstein model, while ex- 


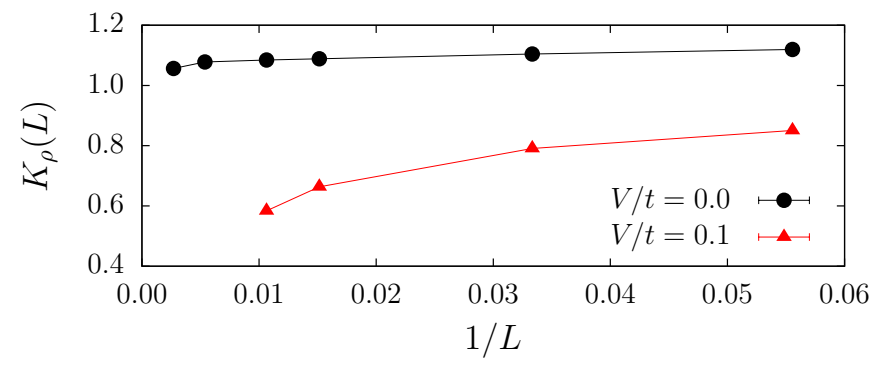

FIG. 17. (Color online) $K_{\rho}(L)$ for the $U-V$ model. Here, $\beta t=$ $2 L, U / t=-4$. Results obtained in the SSE representation.

cluding retardation effects. The aforementioned strongcoupling approximation of Ref. 16 (cf. Sec. IV A) suggests that the low-energy physics of the Holstein model is described by a model of bosonic pairs that hop and interact repulsively. Whereas the critical value for the metalinsulator transition is $V=0$ in the electronic model, the Holstein model supports an extended metallic region.

Figure 17 shows $K_{\rho}(L)$ as a function of $1 / L$. The results for $V=0$ are identical to those in Fig. 14(a), and extrapolate to $K_{\rho}=1$. In contrast, for $V / t=0.1$, corresponding to the insulating phase with long-range charge order, the results for $K_{\rho}(L)$ are compatible with $K_{\rho}=0$.

In Fig. 18, we show results for $\chi_{\rho}(\pi) / L$ in the insulating CDW phase $(V / t=0.1)$. For $U / t=-1$, the spin gap is not resolved in the Luther-Emery phase for $V=0$ (see Fig. 16), which leads to a nonmonotonic finite-size scaling in the CDW phase at $V / t=0.1$. For small $L, \chi_{\rho}(\pi) / L$ decreases, whereas for large $L$ the expected divergence becomes visible. Such nonmonotonic behavior can also be observed for the Holstein model [see Fig. 10(b) and Ref. 10]. The minimum at intermediate $L$ persists for slightly larger $|U / t|$ (but is shifted to smaller $L$ because the spin gap increases with increasing $|U / t|)$, whereas for large $|U / t|$ the gap is large enough to be resolved even for small $L$. For these parameters (e.g., $U / t=-4), \chi_{\rho}(\pi) / L$ is monotonic and exhibits a clear divergence. Because $\chi_{\rho}(\pi) / L$ even diverges (logarithmically) in the metallic phase for $V / t=0$ (see Fig. 16), its qualitative behavior at large $L$ does not permit to distinguish the metallic from the insulating phase.

\section{DISCUSSION}

\section{A. Metallic phase}

Numerical results for the correlation functions reveal that in the spinful Holstein model at half-filling, charge correlations dominate over pairing correlations down to very small values of the electron-phonon coupling $[6,18]$. At the same time, spin correlations are suppressed relative to charge correlations [6]. For a $1 \mathrm{D}$ metallic system, this necessarily implies a gap for spin excitations $[6,40]$, consistent with the observation of $K_{\sigma}<1[6]$.

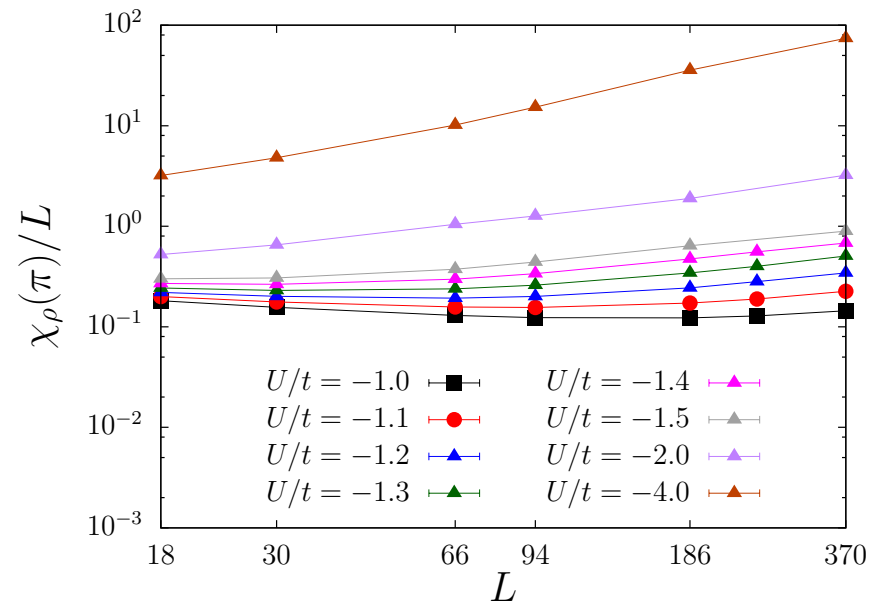

FIG. 18. (Color online) Finite-size scaling of the charge susceptibility for the $U-V$ model. Here, $\beta t=2 L, V / t=0.1$. Results obtained in the SSE representation.

The gap arises from the pairing of electrons into spin singlets (bipolarons) or, in the language of bosonization, from attractive backscattering which is a relevant perturbation of the Tomonaga-Luttinger fixed point.

The spin gap makes the Luther-Emery liquid the relevant fixed point for a low-energy description of the metallic phase, and significantly complicates the analysis of numerical data because a crossover from the Luttinger to the Luther-Emery fixed point takes place as a function of distance. For distances smaller than the inverse spin gap, correlation functions resemble those of a gapless Luttinger liquid, whereas for large distances the corresponding Luther-Emery results are approached. Because the spin gap is small in the metallic phase for typical phonon frequencies, it appears highly nontrivial to reach the correct low-energy fixed point numerically. Importantly, the exponents for charge and pairing correlations are expected to change in the same way when the spin gap is resolved, leaving the conclusion that the metallic phase is a repulsive Luther-Emery liquid unchanged. The fixed-point crossover and the complications in determining the Luttinger parameters and the phase boundary are absent in the spinless Holstein and $t$ - $V$ models.

Assuming that the spin gap in the Holstein model is roughly determined by the corresponding attractive Hubbard interaction $U=-4 \lambda t$, the critical values $\lambda_{\mathrm{c}} \approx 0.25$ (for $\omega_{0} / t=0.5$ ) and $\lambda_{\mathrm{c}} \approx 0.5$ (for $\omega_{0} / t=5$ ) translate into $U / t=-1$ and -2 , respectively. Hence, the results for the attractive Hubbard model in Fig. 15 should be representative of the largest spin gaps in the metallic phase of the Holstein model for typical phonon frequencies, and suggest that the Luther-Emery regime will be hard to reach numerically (e.g., for $U / t=-1, L=370$ sites are not sufficient). If system sizes are too small, correlation functions will not display the expected behavior.

The numerical finding of $K_{\rho}>1$ in the metallic phase has motivated much of the recent work on the Holstein 
model. Initial claims of dominant pairing correlations turned out to be incorrect, and the system instead exhibits dominant charge correlations suggestive of $K_{\rho}<1$. We have shown that the inconsistent values of $K_{\rho}$ cannot be explained by retardation effects $\left(K_{\rho}\right.$ deviates even more from 1 for higher phonon frequencies). Instead, because very similar problems arise in simulations of the attractive Hubbard model, we attribute the complications to backscattering and the spin gap.

\section{B. Low-energy theory}

While theories of metal-insulator transitions in LutherEmery liquids are less controlled than for Luttinger liquids [59], a consistent picture of the spinful Holstein model is in terms of interacting bipolarons that order into a CDW state at $\lambda_{c}$. Such a description was previously suggested for the extended Hubbard model [57], and also emerges from a strong-coupling approximation of the Holstein model [16]. For simplicity, we focus the discussion on hard-core bosons, although bipolarons in the Holstein model can also be extended.

Adopting the bosonic picture, the bosonization and RG methods predict metallic behavior (reflected in a vanishing two-particle electronic charge gap) for $K>1 / 2$ (we drop the index $\rho$ when referring to the bosonic picture). Similar to spinless fermions, a metallic phase with dominant charge correlations and subdominant pairing correlations exists for $1 / 2<K<1$. At $K=1 / 2$, a Mott transition to an insulating CDW state takes place. Clearly, the bosonic picture provides a strong connection to the spinless Holstein model, where the Luttinger liquid to Peierls insulator transition may be understood in terms of a Mott transition of spinless fermions.

The value of $K_{\rho}$ at the Peierls transition in the spinful Holstein model is not known from theory (the assumption of $K_{\rho}=1$ in Ref. 12 seems unjustified because there is no cancellation of interactions at $\lambda_{c}$ ). The transition from a Luther-Emery to a CDW phase was studied for the $U-V$ extended Hubbard model, where the symmetries for $V=0$ imply that the critical value is $K_{\rho}=1$. However, the bosonic low-energy theory of the Holstein model contains an interaction between pairs of electrons, and we therefore expect a different scaling dimension and critical $K$. Additionally, the role of quantum lattice fluctuations needs to be addressed. Indeed, recent functional RG results for the Holstein model [17] confirm the existence of a finite metallic region.

For numerical investigations of the Holstein model, it is important to recognize that the system size has to be sufficiently large to resolve the spin gap in order to allow for a meaningful comparison with the bosonic picture. The electronic Luttinger parameter $K_{\rho}$ may in general not have a simple relation to the $K$ of the bosonic picture. Of particular interest is a numerical calculation of bosonic pairing and density correlation functions. The latter would permit to test the bosonic picture and the corresponding bosonization results (including the transition at $K=1 / 2$ ) quantitatively.

\section{CONCLUSIONS}

We carried out extensive quantum Monte Carlo simulations to resolve conflicts regarding the existence of a metallic phase and the value to the Luttinger parameter $K_{\rho}$ in the one-dimensional spinful Holstein model at half-filling. In addition, we considered the spinless Holstein model and minimal fermionic models that capture the charge-density-wave transition.

First, we showed that the results of Ref. 16 are partly incorrect (likely due to autocorrelations), and should hence not be regarded as evidence for the absence of a metallic phase in the Holstein model, in accordance with recent functional renormalization group results that predict a nonzero critical electron-phonon coupling [17].

Our results for the real-space correlation functions in the metallic phase are consistent with Luttinger liquid physics for the spinless Holstein model, and LutherEmery physics for the spinful Holstein model. In the latter case, spin correlations are suppressed with respect to charge correlations due to the existence of a spin gap, although the gap is typically not fully resolved in numerical simulations. In both models, charge correlations dominate over pairing correlations, which suggests predominantly repulsive interactions.

Given a metallic phase at weak coupling, we investigated in detail how the spin gap manifests itself in finitesize simulations. By comparing results for the spinful and the spinless Holstein model, as well as for fermionic models, we revealed that complications in accurately determining $K_{\rho}$ appear to be generic for spin-gapped phases, but independent of retardation effects. As a function of distance, correlation functions reveal a crossover from Luttinger liquid behavior at short distances to LutherEmery behavior at long distances. A nonzero spin gap also affects the possibility of using the charge susceptibility to track the Peierls transition, which may explain existing deviations between phase boundaries in the literature. Our results and analysis of previous work suggest that even with state-of-the-art numerical methods the paradigmatic spinful Holstein and Holstein-Hubbard models remain challenging.

Finally, our work motivates further numerical and analytical investigations. On the numerical side, it would be useful to find alternative ways to determine the critical value of the Peierls transition, and to measure bosonic correlation functions to verify the low-energy description in terms of bipolarons. On the theoretical side, an improved understanding of the origin of logarithmic corrections to $K_{\rho}$, and of the Mott transition in a generic Luther-Emery liquid would be desirable. 


\section{ACKNOWLEDGMENTS}

We acknowledge computer time at the Jülich Supercomputing Centre, financial support from the DFG via FOR 1807, and valuable discussions with C. Bourbonnais, S. Eggert, S. Ejima, F. Essler, H. Fehske, T. Giamarchi, V. Meden, A. Sandvik, and D. Schuricht.

\section{Appendix A: Scaling of the charge susceptibility}

For $K_{\rho}<1$, the dominant contribution to the equaltime charge correlations in a Luttinger or Luther-Emery liquid has $Q=2 k_{\mathrm{F}}$, with $Q=\pi$ for half-filling. Writing the equal-time $2 k_{\mathrm{F}}$ charge correlations in the form $(-1)^{r} / r^{\alpha}$, and exploiting conformal invariance, we have

$$
\left\langle\hat{n}_{r}(\tau) \hat{n}_{0}(0)\right\rangle \sim \frac{(-1)^{r}}{\left(r^{2}+\tau^{2}\right)^{\alpha / 2}}
$$

for the charge correlation function in Eq. (13). The rescaled charge susceptibility becomes

$$
\frac{\chi_{\rho}(\pi)}{L} \sim \frac{1}{L} \sum_{r} \int_{0}^{\beta} \frac{d \tau}{\left(r^{2}+\tau^{2}\right)^{\alpha / 2}} .
$$

Taking the continuum limit, and regularizing with a short-distance cutoff $a$, we obtain

$$
\frac{\chi_{\rho}(\pi)}{L} \sim \frac{1}{L} \int_{a}^{L} d x \int_{a}^{\beta} d \tau\left(x^{2}+\tau^{2}\right)^{-\alpha / 2} .
$$

Transforming to polar coordinates $(\rho, \phi)$, setting $\beta=L$, and assuming $\alpha<2$ we find

$$
\frac{\chi_{\rho}(\pi)}{L} \sim \frac{2 \pi}{L} \int_{a}^{L} d \rho \rho^{1-\alpha} \sim L^{1-\alpha}\left[1-\left(\frac{a}{L}\right)^{2-\alpha}\right] .
$$

Finally, taking the limit $a \rightarrow 0$, we obtain

$$
\frac{\chi_{\rho}(\pi)}{L} \sim L^{1-\alpha}
$$

1 R. Peierls, Surprises in Theoretical Physics (Princeton University Press, New Jersey, 1979).

2 L. D. Landau, Phys. Z. Sowjetunion 3, 644 (1933).

3 D. M. Edwards, Adv. Phys. 51, 1259 (2002).

4 J. T. Devreese and A. S. Alexandrov, Rep. Prog. Phys. 72, 066501 (2009).

5 T. Holstein, Ann. Phys. (N.Y.) 8, 325 (1959); 8, 343 (1959).

6 M. Hohenadler and F. F. Assaad, Phys. Rev. B 87, 075149 (2013).

7 I. P. Bindloss, Phys. Rev. B 71, 205113 (2005).

${ }^{8}$ H. Bakrim and C. Bourbonnais, Phys. Rev. B 76, 195115 (2007).

9 R. T. Clay and R. P. Hardikar, Phys. Rev. Lett. 95, 096401 (2005).

10 R. P. Hardikar and R. T. Clay, Phys. Rev. B 75, 245103 (2007).

11 H. Fehske, G. Hager, and E. Jeckelmann, Europhys. Lett. 84, 57001 (2008).

12 S. Ejima and H. Fehske, J. Phys. Conf. Ser. 200, 012031 (2010).

13 R. J. Bursill, R. H. McKenzie, and C. J. Hamer, Phys. Rev. Lett. 80, 5607 (1998).

14 M. Hohenadler, G. Wellein, A. R. Bishop, A. Alvermann, and H. Fehske, Phys. Rev. B 73, 245120 (2006).

15 S. Ejima and H. Fehske, Europhys. Lett. 87, 27001 (2009).

16 J. E. Hirsch and E. Fradkin, Phys. Rev. B 27, 4302 (1983).

17 H. Bakrim and C. Bourbonnais, Phys. Rev. B 91, 085114 (2015).

18 K.-M. Tam, S.-W. Tsai, D. K. Campbell, and A. H. Castro Neto, Phys. Rev. B 75, 161103 (2007).

19 K.-M. Tam, S.-W. Tsai, and D. K. Campbell, Phys. Rev. B 84, 165123 (2011).

20 M. Tezuka, R. Arita, and H. Aoki, Phys. Rev. Lett. 95, 226401 (2005).
21 M. Tezuka, R. Arita, and H. Aoki, Phys. Rev. B 76, 155114 (2007).

22 A. Luther and V. J. Emery, Phys. Rev. Lett. 33, 589 (1974).

23 W. von der Linden, E. Berger, and P. Valášek, J. Low Temp. Phys. 99, 517 (1995).

24 A. N. Rubtsov, V. V. Savkin, and A. I. Lichtenstein, Phys. Rev. B 72, 035122 (2005).

${ }^{25}$ F. F. Assaad, Phys. Rev. B 78, 155124 (2008).

26 M. Hohenadler, H. Fehske, and F. F. Assaad, Phys. Rev. B 83, 115105 (2011).

27 M. Hohenadler, F. F. Assaad, and H. Fehske, Phys. Rev. Lett. 109, 116407 (2012).

28 M. Hohenadler and F. F. Assaad, J. Phys.: Condens. Matter 25, 014005 (2013).

29 F. F. Assaad and T. C. Lang, Phys. Rev. B 76, 035116 (2007).

30 E. Gull, A. J. Millis, A. I. Lichtenstein, A. N. Rubtsov, M. Troyer, and P. Werner, Rev. Mod. Phys. 83, 349 (2011).

31 F. F. Assaad, in DMFT at 25: Infinite Dimensions, Modeling and Simulation, edited by E. Pavarini, E. Koch, D. Vollhardt, and A. Lichtenstein (Forschungszentrum Jülich Zentralbibliothek, Verlag, 2014) Chap. 7.

32 O. Syljuasen and A. W. Sandvik, Phys. Rev. E 66, 046701 (2002).

33 To avoid a sign problem in the SSE representation, instead of the particle-hole symmetric form of the interaction in Eq. (1) for which $\mu=0$ corresponds to half-filling, we used $-g \sum_{i} \hat{Q}_{i} \hat{n}_{i}$ and a chemical potential $\mu=4 \lambda t$.

34 A. Sandvik, R. Singh, and D. Campbell, Phys. Rev. B 56, 14510 (1997).

35 P. Sengupta, A. W. Sandvik, and D. K. Campbell, Phys. Rev. B 65, 155113 (2002). 
${ }^{36}$ H. Fehske, G. Wellein, A. Weisse, F. Gohmann, H. Buttner, and A. R. Bishop, Physica B 312-313, 562 (2002).

37 H. Fehske, A. P. Kampf, M. Sekania, and G. Wellein, Eur. Phys. J. B 31, 11 (2003).

${ }^{38}$ H. Fehske, G. Wellein, G. Hager, A. Weiße, and A. R. Bishop, Phys. Rev. B 69, 165115 (2004).

39 E. Jeckelmann, C. Zhang, and S. R. White, Phys. Rev. B 60, 7950 (1999).

40 J. Voit, Eur. Phys. J. B 5, 505 (1998).

41 T. Giamarchi, Quantum Physics in One Dimension (Clarendon Press, Oxford, 2004).

42 M. Weber, F. F. Assaad, and M. Hohenadler, Phys. Rev. B 91, 245147 (2015).

43 M. Hohenadler, D. Neuber, W. von der Linden, G. Wellein, J. Loos, and H. Fehske, Phys. Rev. B 71, 245111 (2005).

44 J. Voit, Rep. Prog. Phy. 58, 977 (1995).

45 J. Cardy, Scaling and Renormalization in Statistical Physics (Cambridge University Press, Cambridge, 1996).

46 T. Giamarchi and H. J. Schulz, Phys. Rev. B 39, 4620 (1989).

47 I. Affleck, D. Gepner, H. J. Schulz, and T. Ziman, J. Phys. A-Math. Gen. 22, 511 (1989).

48 S. Lukyanov, Nuclear Physics B 522, 533 (1998).

49 In contrast to the spinless $t-V$ model discussed in Sec. V A, the values of $K_{\rho}$ reported in Ref. 15 do not exactly match the power-law exponents of the correlation functions. This suggests that the finite-size extrapolation of $K_{\rho}(L)$ is less reliable for the spinless Holstein model.

50 A. W. Sandvik and D. K. Campbell, Phys. Rev. Lett. 83, 195 (1999).

51 P. Sengupta, A. W. Sandvik, and D. K. Campbell, Phys. Rev. B 67, 245103 (2003).

52 A. S. Sandvik, L. Balents, and D. K. Campbell, Phys. Rev. Lett. 92, 236401 (2004).

53 H. Bethe, Z. Phys. B 71, 205 (1931).

54 C. Karrasch and J. E. Moore, Phys. Rev. B 86, 155156 (2012).

55 S. Qin, M. Fabrizio, L. Yu, M. Oshikawa, and I. Affleck, Phys. Rev. B 56, 9766 (1997).

56 S. Ejima, F. Gebhard, and S. Nishimoto, Europhys. Lett.) 70, 492 (2005).

57 V. J. Emery, in Highly conducting one-dimensional solids, edited by J. T. Devreese (Plenum Press, New York, 1979) p. 247.

58 H. J. Schulz, Phys. Rev. Lett 64, 2831 (1990).

59 J. Voit, Phys. Rev. B 45, 4027 (1992).

60 S. A. Söffing, I. Schneider, and S. Eggert, Europhys. Lett. 101, 56006 (2013).

61 R. T. Clay, A. W. Sandvik, and D. K. Campbell, Phys. Rev. B 59, 4665 (1999). 